\title{
KRÜMMUNGSEIGENSCHAFTEN KONVEXER FLÄCHEN *
}

\author{
VON \\ HERBERT BUSEMANN und WILLY FELLER \\ in KOPENHAGEN.
}

\section{Inhalt.}

Einleitung . . . . . . . . . . . . . . . . . . . . . . 1

$\S$ I. Vorbemerkungen über Kurven . . . . . . . . . . . . . . . . . . 4

$\S$ 2. Der Meusniersche Satz . . . . . . . . . . . . . . . . . . . . . 8

$\S$ 3. Die Krümmungen der Normalschnitte im einzelnen Flächenpunkt . . . 13

$\S$ 4. Fast überall vorhandene Krümmungseigenschaften der Normalschnitte . 23

$\$$ 5. Über den Nabelpunktsatz . . . . . . . . . . . . . . . . . . . . 30

$\S 6$. Beispiel einer Fläche, die bis auf eine Nullmenge aus lauter Kugelstïcken desselben Radius besteht . . . . . . . . . . . . . . . . . . . . 34

$\S$ 7. Kürzeste Linien . . . . . . . . . . . . . . . . . . . . . . . . 40

\section{Kinleitung.}

Die vorliegende Arbeit sucht die Elemente der Differentialgeometrie der konvexen Flächen ohne die üblichen Regularitätsannahmen zu entwickeln. Dabei treten vielfach zwangsläufig rein geometrische Überlegungen an Stelle der sonstigen analytischen, wodurch sich die Methode derjenigen von HJELmsiev in seinen "Grundlag for Fladernes Geometri» ${ }^{1}$ nähert. Weniger Beziehungen bestehen zu den Arbeiten von Bouligand und seiner Schule, da in diesen - soweit sie unsren Gegenstand betreffen - mehr geometrische Formulierungen der analytischen Vorgänge als unmittelbare geometrische Ansätze gegeben werden.

1 Mém. Acad. Roy. Sc. Let. Danemark, Copenhague, (7) XII, (19I4).

1-35150. Acta mathematica. 66. Imprimé le 26 juillet 1935. 
Unsre Hauptthemata sind: Analoga der Sätze von Meusnier, Olinde Rodrigues und Euler; der Satz, dass die Kugel die einzige geschlossene Fläche mit lauter Nabelpunkten ist; schliesslich die Differentialeigenschaften der kürzesten Linien.

In $\S$ I stellen wir einige Definitionen und Eigenschaften von Kurven zusammen. Der $\S 2$, welcher auch für nichtkonvexe Flächen gilt, befasst sich mit dem Meusnierschen Satz. Die Einführung der folgenden Begriffsbildung ist dabei zweckmässig. Die Ebene $\Pi$ heisst im Punkte $P$ der Fläche $\boldsymbol{\Phi}$ Tangentialebene im scharfen Sinne (paratingent plan bei Bouligand), wenn für jede gegen $P$ konvergierende Sehnenfolge $\overline{Q_{n} R_{n}}$ sämtliche Grenzlagen der Geraden $\underline{Q_{n} R_{n}}$ in $I$ liegen. Beispielsweise ist jede Tangentialebene einer konvexen Fläche zugleich Tangentialebene im scharfen Sinne. Hat in einem Punkte $P$, in welchem die Fläche eine Tangentialebene im scharfen Sinne besitzt, ein ebener Schnitt (die Tangentialebene immer ausgenommen) eine Krümmung ${ }^{2}$, so haben alle ebenen Schnitte mit derselben Tangente in $P$ ebenfalls eine Krümmung, und die zugehörigen Krümmungskreise liegen auf einer Kugel. Dies bildet eine unwesentliche, aber für konvexe Flächen wichtige Verschärfung des Meusnierschen Satzes in der Formulierung von HJecmsLev. ${ }^{3}$ - Der bekannte Zusammenhang der Krümmung einer Flächenkurve mit der ihrer Projektion auf die Tangentialebene gilt auch bereits unter diesen allgemeinen Voraussetzungen.

In $\$ 3$ werden die Krümmungen der Normalschnitte einer konvexen Fläche in einem Punkte $P$ untersucht, in dem die Fläche eine Tangentialebene hat. Trägt man die Wurzel aus dem kleinsten (einseitigen) Krümmungsradius jedes Normalschnitts auf der zugehörigen Halbtangente von $P$ aus ab, so beschreibt der Endpunkt eine Kurve, die wir die untere Indikatrix von $P$ nennen. Diese ist stets eine konvexe Kurve (die freilich ganz im Unendlichen liegen kann). Es folgen Aussagen über die analog zu definierende obere Indikatrix. Von einer Indikatrix schlechthin sprechen wir, wenn beide Kurven zusammenfallen. Jede konvexe Kurve, die $P$ im Innern oder am Rande enthält, kann als Indikatrix des Flächenpunktes $P$ auftreten.

Die konvexe Fläche möge nun in einer Umgebung von $P$ eine Tangential-

2 Der Krämmungsmittelpunkt ist hierbei nicht aufzufassen als Grenzlage des Schnittpunktes benachbarter Kurvennormalen, sondern als Grenzlage der Mittelpunkte der Kreise, die in $P$ die Kurve berühren, und durch einen benachbarten Kurvenpunkt gehen.

${ }^{3}$ a. a. 0 . - Neuerdings hat auch Bouligand den Meusniersehen Satz unter geringeren als den klassischen, aber weit schärferen als den Hjelmslevschen Voraussetzungen bewiesen. Vgl. im Folgenden S. 11. 
ebene und in $P$ selbst ausserdem eine Indikatrix besitzen; wir betrachten die durch parallele Normalen vermittelte Abbildung der Fläche auf die Einheitskugel. Ist $Q$ ein Punkt der Indikatrix von $P$, in dem diese eine Tangente $\tau$ hat, so hat das sphärische Bild jedes ebenen Flächenschnitts mit der Tangente $P Q$ im Bildpunkt von $P$ eine Tangente, und diese steht senkrecht auf $\tau$. Die Schnittgeraden benachbarter Tangentialebenen längs dieser Flächenschnitte haben daher bei Annäherung an $P$ eine Grenzlage, und diese ist parallel zu $\tau$. Dies entspricht dem Satze über konjugierte Richtungen im regulären Falle: Wenn die Indikatrix keine Ecken hat, so gibt es zu jeder Richtung durch $P$ eine konjugierte; diese Beziehung kann sogar symmetrisch sein, ohne dass die Indikatrix eine Ellipse ist. Zu den Richtungen $P Q$, in denen der Radiusvektor der Indikatrix einen Extremwert annimmt, ist die Senkrechte konjugiert; in diesen Richtungen (deren es im allgemeinem beliebig viele geben kann) gilt die Formel von Olinde Rodrigues für die Hauptkrümmungsrichtungen.

In $\S 4$ verzichten wir darauf, in jedem Punkte gültige Aussagen über die Krümmungen der Normalschnitte zu machen, und erhalten statt dessen schärfere Sätze, die aber nur "fast überall" (d. h. mit Ausnahme einer Menge vom Masse Null) statthaben. Sätze dieses Typus lassen sich natürlich nur mit Hilfe der Theorie der reellen Funktionen gewinnen. Konvexe Flächen haben fast überall eine Tangentialebene ${ }^{4}$, und konvexe Kurven haben fast überall eine endliche Krümmung. ${ }^{5}$ Mit Hilfe des Meusnierschen Satzes und der Resultate des § 3 schliesst man hieraus, dass in fast allen Punkten der Fläche sämtliche ebenen Schnitte eine endliche Krümmung haben. Darüber hinaus gilt in fast allen Punkten der Eulersche Satz, d. h. die Indikatrix ist fast überall ein Kegelschnitt mit dem betreffenden Punkt als Mittelpunkt. Es ist vielleicht von Interesse, dass die letzteren Punkte analytisch so charakterisiert sind: Man betrachtet ein Flächenstück $\boldsymbol{\Phi}$, dass sich eindeutig auf eine Ebene $\Pi$ projiziert und führt in $I I$ auf alle möglichen Arten kartesische Koordinatensysteme $x, y$ ein. Stellt man in einem dieser Systeme $\Phi$ in der Form $z=f(x, y)$ dar, so ist $f(x, y)$ von beschränkter Schwankung und daher fast überall nach Rechtecken differenzierbar. ${ }^{6}$ Es handelt

4 Vgl. T. Bonnesen und W. Fenchel: Theorie der konvexen Körper; Ergebnisse der Math., III, I, Berlin I934, S. I3. - Wir beziehen uns, soweit möglich, auf diesen Bericht, und zitieren ihn zur Abkürzung mit B. F.

5 B. F. S. 144.

${ }^{6}$ d. h. in fast allen Punkten existiert

$$
\lim _{n \rightarrow \infty} \frac{f\left(x+h_{n}, y+k_{n}\right)+f\left(x-h_{n}, y-k_{n}\right)-f\left(x+h_{n}, y-k_{n}\right)-f\left(x-h_{n}, y+k_{n}\right)}{4 h_{n} k_{n}}
$$


sich dann um diejenigen Punkte, in denen I) alle ebenen Schnitte endliche Krïmmungen besitzen, und 2) alle diese Funktionen $f(x, y)$ gleichzeitig differenzierbar sind.

Im $\S 5$ wird ohne weitere Regularitätsannahmen bewiesen, dass ein differenzierbares Flächenstück, das überall aus Nabelpunkten besteht, ein Kugelstück sein muss. Es liegt die Frage nahe, ob es hier genügt vorauszusetzen, dass das Flächenstück nur fast ïberall aus Nabelpunkten besteht. Dieses ist unter gewissen Voraussetzungen der Fall, etwa wenn die Krümmungen der Normalschnitte beschränkt sind, oder wenn die ersten Flächenableitungen totalstetig sind. Dagegen zeigt der $\$ 6$, dass der Satz ohne solche Voraussetzungen nicht richtig ist. Wir geben nämlich eine geschlossene differenzierbare konvexe Fläche an, von der fast alle Punkte Nabelpunkte derselben Krümmung sind, und die doch keine Kugel ist; die Krümmung kann dabei auch gleich Null sein. Diese Fläche ist gleichzeitig ein Beispiel dafür, dass die sphärische Abbildung differenzierbarer konvexer Flächen nicht totalstetig zu sein braucht, d. h. dass Nullmengen auf der Fläche in Mengen positiven Masses auf der Kugel übergehen können. Dass das bei beschränkten Krümmungen der Normalschnitte nicht vorkommen kann, zeigen wir schon vorher durch Anwendung einer in $\$ 5$ vorkommenden Beziehung.

Der letzte Paragraph ist unabhängig von den $\$ \$ 3-6$. Nach LeBesgue ${ }^{7}$ gibt es auf jeder konvexen Fläche zwischen je zwei Punkten eine kürzeste Verbindung. In $\S 7$ wird gezeigt, dass eine solche kürzeste Linie in jedem Punkte $P$ (die Eckpunkte inbegriffen), in dem die Fläche eine Tangentialebene hat, differenzierbar ist und eine Schmiegebene besitzt, die durch die Flächennormale geht. Die Projektion der Linie auf die Tangentialebene hat in solchen Punkten die Krïmmung Null, selbst wenn die Normalschnitte (und also auch die kürzeste Linie) keine Krïmmung haben.

§ I.

\section{Vorbemerkungen ïber Kurven.}

Alle im Folgenden vorkommenden geometrischen Gebilde liegen im dreidimensionalen Euklidischen Raum. Grosse lateinischen Buchstaben bezeichnen wobei $h_{n}$ und $h_{n}$ wei Nullfolgen sind, die nur der Einschränkung

unterworfen werden.

$$
\circ<\underline{\lim } \frac{h_{n}}{k_{n}} \leq \overline{\lim } \frac{h_{n}}{k_{n}}<\infty
$$

7 Annali di Matematica (3), VII (1902). Vgl. auch O. BoIzA: Variationsrechnung, Leipzig und Berlin 1909, S. 422 . 
stets Punkte, $\overline{P Q}$ die abgeschlossene Strecke von $P$ nach $Q$, und $\underline{P Q}$, wenn $P \neq Q$, die Gerade durch $P$ und $Q$. Wenn $R$ nicht auf der Geraden $P Q=t$ liegt, bedeute ferner $\underline{P Q R}=\underline{R t}$ die Ebene durch $R$ und $t$. Die Entfernung zweier Punktmengen $\alpha$ und $\beta$ wird mit $e(\alpha, \beta)$ bezeichnet.

Es ist bequem, einige gewöhnlich nur für Kurven definierte Begriffe auf konvergente Punktfolgen auszudehnen. Wenn für die gegen $P$ konvergierende Punktfolge $\left\{P_{n}\right\}$ die Geraden $P P_{n}$ konvergieren, heisse die Grenzgerade $t$ Tangente von $\left\{P_{n}\right\}$. Es sei dann $F_{P_{n}}$ der Fusspunkt des Lotes von $P_{n}$ auf $t$; wir nennen die Grösse $\sigma_{n}=e\left(P_{n}, F_{P_{n}}\right)$ den Normalen- und $\tau_{n}=e\left(F_{P_{n}}, P\right)$ den Tangentenabschnitt von $P_{n}$ auf $t$. Es ist

$$
\lim \frac{\sigma_{n}}{\tau_{n}}=0
$$

Der Kreis durch die Punkte $P$ und $P_{n}$ mit der Tangente $t$ in $P$ heisse Näherungskreis von $P_{n}$ bezüglich des Linienelementes $(P, t)$. Sein Radius ist

$$
\varrho\left(P_{n}\right)=\frac{e^{2}\left(P, P_{n}\right)}{2 e\left(P_{n}, F_{P_{n}}\right)}=\frac{\sigma_{n}^{2}+\tau_{n}^{2}}{2 \sigma_{n}},
$$

wobei für $\sigma_{n}=$ o die rechte Seite das Symbol $\infty$ darstellt. Wenn $\varrho=\lim \varrho\left(P_{n}\right)$ existiert, nennen wir ihn den Kriimmungsradius der Punktfolge $\left\{P_{n}\right\}$. Aus (I) und (2) findet man für den Krümmungsradius:

$$
\varrho=\lim \frac{\tau_{n}^{2}}{2 \sigma_{n}} .
$$

Die Zahl I/ $/$ bzw. 0 im Falle $\varrho=\infty$ heisse die Krümmung von $\left\{\boldsymbol{P}_{n}\right\}$.

Wenn die konvergente Punktfolge $P_{n} \rightarrow P$ eine Tangente $t$ hat und die Ebenen $\underline{P_{n} t}$ konvergieren, nennen wir deren Grenzlage die Schmiegebene von $\left\{P_{n}\right\}$. Wir sprechen ferner von einer Schmiegebene auch dann, wenn $\left\{P_{n}\right\}$ zwar keine Tangente hat, aber alle Teilfolgen mit 'Tangente dieselbe Schmiegebene besitzen; das ist z. B. bei allen ebenen Kurven der Fall. Hat eine Punktfolge $\left\{P_{n}\right\}$ sowohl eine Schmiegebene $I I$ als auch eine Krümmung I/g, so hat die Folge der Fusspunkte. von $P_{n}$ auf $I$ dieselbe Krümmung. Sind $\Pi^{\prime}$ und $\Pi^{\prime \prime}$ zwei zueinander senkrechte Ebenen durch $t$, so haben die Folgen der Fusspunkte von $P_{n}$ auf diesen Ebenen ebenfalls Krümmungen $\mathrm{I} / \varrho^{\prime}$ und $\mathrm{I} / \varrho^{\prime \prime}$, und es ist

$$
\frac{\mathrm{I}}{\varrho^{2}}=\frac{\mathrm{I}}{\varrho^{\prime 2}}+\frac{\mathrm{I}}{\varrho^{\prime \prime 2}} .
$$


Nun sei $\varkappa$ das topologische Bild einer abgeschlossenen Strecke, und $P$ ein Endpunkt desselben. Wenn alle gegen $P$ konvergierenden Punktfolgen auf $x$ eine (und daher dieselbe) Tangente $t$ haben, so heisse $t$ Tangente an $x$ in $P$. Wenn für jede gegen $P$ konvergierende Sehnenfolge $\overline{Q_{n} R_{n}}$ von $x$ die Geraden $Q_{n} R_{n} \rightarrow t$, so nennen wir $t$ Tangente im scharfen Sinne.

Macht man $P$ zum Ursprung und die Tangente im scharfen Sinne $t$ zur $x$-Achse eines Koordinatensystems, so hat $x$ in einer Halbumgebung des Ursprungs, etwa für $x \geq 0$, eine eindeutige Darstellung der Form $y=f(x), z=g(x)$. Wenn die $x$-Achse im Ursprung nur gewöhnliche Tangente ist, so braucht eine solche Darstellung nicht zu existieren. Dass die $x$-Achse im Ursprung Tangente im scharfen Sinne ist, drückt sich analytisch durch die Beziehungen

$$
\lim _{\substack{x \rightarrow+0 \\ h \rightarrow 0}} \frac{f(x+h)-f(x)}{h}=\lim _{\substack{x \rightarrow+0 \\ h \rightarrow 0}} \frac{g(x+h)-g(x)}{h}=0
$$

aus. Die Differenzenquotienten von $f(x)$ und $g(x)$ sind daher in einer Umgebung des Nullpunktes beschränkt, so dass dort die Ableitungen $f^{\prime}(x)$ und $g^{\prime}(x)$ fast überall existieren. Für jede Nullfolge $\left\{x_{n}\right\}$, für die $f^{\prime}\left(x_{n}\right)$ und $g^{\prime}\left(x_{n}\right)$ existieren, gilt $f^{\prime}\left(x_{n}\right) \rightarrow 0$ und $g^{\prime}\left(x_{n}\right) \rightarrow$ o. Diese Bedingungen sind auch hinreichend dafür, dass die $x$-Achse im Nullpunkt Tangente im scharfen Sinne ist. ${ }^{8}$

$x$ habe wieder in $P$ die Tangente $t$ (nicht notwendig im scharfen Sinne). Wir betrachten alle gegen $P$ konvergierenden Punktfolgen auf $x$, die mit einer Krümmung versehen sind. Die untere (obere) Grenze aller so erhaltenen Krümmungen nennen wir die untere (obere) Krïmmung von $\varkappa$ in $P$. Entsprechend werden die Begriffe: Krümmung, Krümmungsradien und Schmiegebene erklärt.

Jeder innere Punkt $Q$ zerlegt $x$ in zwei Teilbogen $x_{r}$ und $x_{l}$, welche wir die rechte bzw. die linke Seite von $Q$ auf $x$ nennen wollen. Wenn $x_{r}$ und $x_{l}$ in $Q$ Tangenten haben, nennen wir diese die rechte bzw. linke Tangente von $x$ in $Q$; wenn beide zusammenfallen, sprechen wir von einer Tangente schlechthin. In analoger Weise werden die übrigen Begriffe definiert.

Wir betrachten nun eine ebene Kurve $x$, die durch die Gleichung $y=f(x)$ dargestellt ist. Wenn $x$ in $x=x_{0}$ eine rechte Tangente besitzt, so existiert die rechte Ableitung $f^{r}\left(x_{0}\right)$, und man findet für die rechte obere und untere Krümmung die Werte 


$$
\frac{2}{\sqrt{\mathrm{I}+\left[f^{(r)}\left(x_{0}\right)\right]^{2^{3}}}} \varlimsup_{h \rightarrow+0} \frac{\mathrm{I}}{h}\left\{\frac{f\left(x_{0}+h\right)-f\left(x_{0}\right)}{h}-f^{r}\left(x_{0}\right)\right\} \text {. }
$$

Man sieht hieraus, dass $x$ in $x=x_{0}$ selbst bei stetiger erster Ableitung $f^{\prime}(x)$ eine Krümmung haben kann, ohne dass eine zweite Ableitung $f^{\prime \prime}\left(x_{0}\right)$ existiert. ${ }^{9}$ Ein Beispiel dafür liefert $y=x^{3} \sin \frac{\mathrm{I}}{x}$ im Nullpunkt. Wenn dagegen die zweite rechte Ableitung $f^{r r}\left(x_{0}\right)$ existiert, so besitzt $x$ in $x=x_{0}$ eine rechte Krümmung ${ }^{10}$, und zwar ist diese gleich

$$
\frac{I}{\varrho_{0}}=\frac{f^{r r}\left(x_{0}\right)}{\sqrt{\mathrm{I}+\left[\bar{f}^{r}\left(x_{0}\right)\right]^{3}}} .
$$

Insbesondere hat $x$, wenn $f^{\prime \prime}\left(x_{0}\right)$ existiert, in $x=x_{0}$ eine Krümmung schlechthin. Indem man dann (5) und (6) auf die rechte und auf die linke Krümmung anwendet, findet man

$$
f^{\prime \prime}\left(x_{0}\right)=\lim _{h \rightarrow 0} \frac{f\left(x_{0}+h\right)+f\left(x_{0}-h\right)-2 f\left(x_{0}\right)}{h^{2}} .
$$

Bei konvexen Kurven liegen die Verhältnisse besonders einfach. Es gibt hier in jedem Punkt eine rechte und eine linke Tangente, und diese sind monoton. Daher hat die Kurve überall, mit Ausnahme höchstens abzählbar vieler Punkte, eine (zweiseitige) Tangente; diese ist stets zugleich Tangente im scharfen Sinne. Die Monotonität der Tangenten hat zur Folge, dass fast ïberall auch eine zweite Ableitung ${ }^{11}$ der die Kurve darstellenden Funktion existiert. Wichtig ist, dass zum Unterschiede gegen nichtkonvexe Kurven hier aus der. Existenz der rechten (linken) Krümmung die der zweiten rechten (linken) $A b$ leitung folgt. ${ }^{12}$

Schliesslich sei noch der Blaschkesche Auswahlsatz ${ }^{13}$ erwähnt: Aus einer gleichmässig beschränkten Folge konvexer Kwrven $\left\{x_{n}\right\}$ lässt sich eine Teilfolge $\left\{x_{v_{n}}\right\}$

${ }^{9}$ Nur wenn man den Krümmungsradius vermöge der Grenzlage des Schnittpunktes benachbarter Normalen definiert, ist die Existenz der Krümmung gleichbedeutend mit der Existenz der zweiten Ableitung. Kopenhagen.

${ }^{10}$ vgl. B. Jessen: Om konvekse Kurvers Krumning. Matematisk Tidsskrift B, I929,

${ }^{11}$ d. h. genauer: Die Ableitungen der rechten und der linken Ableitung stimmen ausserhalb eine Menge vom Mass $\circ$ überein.

I2 vgl. JESSEN, a. a. $O$.

13 vgl. B. F. S. 34 . 
auswählen, welche gegen eine (konvexe) Kurve $x$ konvergiert. Dabei konvergieren die Kurven mit ihren Tangenten im folgenden Sinne: Es sei $P_{n}$ ein Punkt auf $x_{v_{n}}$, und $P_{n}$ konvergiere gegen einen Punkt, in welchem $x$ eine Tangente hat. Dann konvergieren die Stützgeraden von $x_{\nu_{n}}$ in $P_{n}$ gegen diese Tangente.

\section{$\S 2$.}

\section{Der Meusniersche Satz.}

Es sei $\Phi$ ein topologisches Bild der Kreisscheibe, und $P$ ein innerer Punkt von $\Phi$. Eine Ebene $I I$ heisse im Punkte $P$ Tangentialebene von $\Phi$ im scharfen Sinne, wenn für jede gegen $P$ konvergierende Sehnenfolge $\overline{Q_{n} R_{n}}$ von $\Phi$ sämtliche Grenzlagen der Geraden $Q_{n} R_{n}$ in $\Pi$ liegen. Hat $\Phi$ in $P$ eine Tangentialebene im scharfen Sinne, und steht diese nicht senkrecht zur $x, y$-Ebene, so kann man $\Phi$ in einer Umgebung von $P$ eindeutig in der Form $z=f(x, y)$ darstellen. Für eine so dargestellte Fläche kann man die geometrische Definition auch folgendermassen formulieren: $\Phi$ hat in $\left(x_{0}, y_{0}\right)$ dann und nur dann eine Tangentialebene im scharfen Sinne, wenn die Limites

(I)

$$
\lim _{\substack{(x, y) \rightarrow\left(x_{0}, y_{0}\right) \\ h \rightarrow 0}} \frac{f(x+h, y)-f(x, y)}{h}=f_{x}\left(x_{0}, y_{0}\right),
$$

$$
\lim _{\substack{(x, y) \rightarrow\left(x_{0}, y_{0}\right) \\ k \rightarrow 0}} \frac{f(x, y+k)-f(x, y)}{k}=f_{y}\left(x_{0}, y_{0}\right)
$$

existieren. Hat insbesondere $\Phi$ in jedem Punkte eine Tangentialebene, so ist diese dort und nur dort Tangentialebene im scharfen Sinne, wo sie stetig ist.

Der Vollständigkeit halber geben wir noch an, was die Tangentialebene im scharfen Sinne für die Funktion $f(x, y)$ bedeutet. Wir werden aber von dem folgenden Kriterium keinen Gebrauch machen:

Die Fläche $z=f(x, y)$ hat in $\left(x_{0}, y_{0}\right)$ dann und nur dann eine nicht zur. $x, y$-Ebene senkrechte Tangentialebene im scharfen Sinne, wenn: I) $f_{x}\left(x_{0}, y_{0}\right)$ und $f_{y}\left(x_{0}, y_{0}\right)$ existieren, 2) es eine Umgebung von $\left(x_{0}, y_{0}\right)$ gibt, in der $f(x, y)$ fïr fast alle Werte der einen Veränderlichen totalstetig in der anderen ist ${ }^{\mathbf{1 4}}$, und 3) die

${ }^{14}$ Nach LeBksque ist $g(x)$ in einem Intervall totalstetig, wenn darin $g^{\prime}(x)$ fast überall existiert und für jedes Teilintervall

ist.

$$
\int_{x_{0}}^{x_{1}} g^{\prime}(x) d x=g\left(x_{1}\right)-g\left(x_{0}\right)
$$


daher fast ïberall existierenden Ableitungen $f_{x}(x, y)$ und $f_{y}(x, y)$ in $\left(x_{0}, y_{0}\right)$ stetig sind.

Beweis. a) Wenn es in $\left(x_{0}, y_{0}\right)$ eine Tangentialebene im scharfen Sinne gibt, so sind die in ( $\mathrm{I}$ ) vorkommenden Differenzenquotienten in einer Umgebung von $\left(x_{0}, y_{0}\right)$ beschränkt. Daher ist $f(x, y)$ als Funktion einer Veränderlichen sogar für alle Werte der anderen totalstetig. ${ }^{15}$ Die Stetigkeit der partiellen $\mathbf{A b}$ leitungen in $\left(x_{0}, y_{0}\right)$ ergibt sich aus $(\mathrm{r})$.

b) Die drei Bedingungen seien erfüllt. Wir haben zu zeigen, dass für alle Folgen $\left(x_{n}, y_{n}\right) \rightarrow\left(x_{0}, y_{0}\right)$ und $h_{n} \rightarrow$ o, für die

$$
\lim _{n \rightarrow \infty} \frac{f\left(x_{n}+h_{n}, y_{n}\right)-f\left(x_{n}, y_{n}\right)}{h_{n}}
$$

existiert, dieser Grenzwert gleich $f_{x}\left(x_{0}, y_{0}\right)$ ist. Dazu wählen wir $y_{n}^{\prime}$ so nahe an $y_{n}$, dass die Folge

$$
\frac{f\left(x_{n}+h_{n}, y_{n}^{\prime}\right)-f\left(x_{n}, y_{n}^{\prime}\right)}{h_{n}}
$$

denselben Grenzwert hat, wie (2), und derart, dass $f\left(x, y_{n}^{\prime}\right)$ totalstetig ist. Dann ist

$$
f\left(x_{n}+h_{n}, y_{n}^{\prime}\right)-f\left(x_{n}, y_{n}^{\prime}\right)=\int_{x_{n}}^{x_{n}+h_{n}} f_{x}\left(x, y_{n}^{\prime}\right) d x=h_{n} \Delta_{n}
$$

wo $A_{n}$ eine Zahl ist, die zwischen dem Maximum und dem Minimum von $f_{x}\left(x, y_{n}^{\prime}\right)$ im Integrationsintervall liegt. Wegen der dritten Bedingung ist wirklich

$$
\lim \frac{f\left(x_{n}+h_{n}, y_{n}^{\prime}\right)-f\left(x_{n}, y_{n}^{\prime}\right)}{h_{n}}=\lim \Delta_{n}=f_{x}\left(x_{0}, y_{0}\right)
$$

Wir betrachten noch speziell die konvexen Flächen. Hier umhüllen die Stützebenen in einem Punkte $P$ stets einen konvexen Halbkegel, dessen Erzeugende die Halbtangenten sämtlicher ebener Flächenschnitte in $P$ sind. Nun liegt die Fläche ganz auf einer Seite jeder ihrer Stützebenen, so dass jede Grenzlage von Stützebenen wieder eine Stützebene ist. Andrerseits gibt es zu jeder Flächensehne eine parallele Stützebene, woraus man unmittelbar schliesst, dass jede Tangentialebene einer konvexen Fläche zugleich Tangentialebene im scharfen Sinne ist.

is vgl. etwa H. Lebesgue: Leçons sur l'Intégration, Paris I928, S. 77 und I83.

2-35150. Acta mathematica. 66. Imprimé le 26 juillet 1935. 
Der Begriff der Tangentialebene im scharfen Sinne liefert die naturgemässen Voraussetzungen des Meuswierschen Satzes:

Die Fläche $\Phi$ habe im Punkte $P$ im scharfen Sinne die Tangentialebene $\Pi$. Es seien $P_{n} \rightarrow P$ und $P_{n}^{\prime} \rightarrow P$ zwei Folgen von Flächenpunkten mit derselben Tangente $t$ und mit Schmiegebenen, die gegen die Flächennormale in $P$ um $\vartheta \neq \frac{\pi}{2}$ und $\vartheta^{\prime} \neq \frac{\pi}{2}$ geneigt sind. Sind dann die Winkel der Verbindungslinien $\underline{P}_{n} \boldsymbol{P}_{n}^{\prime}$ mit $t$ nach unten beschränkt, und hat $\left\{P_{n}\right\}$ die Krümmung $\frac{\mathrm{I}}{\varrho}$, so besitzt $\left\{P_{n}^{\prime}\right\}$ die Krümmung $\frac{\mathrm{I}}{\varrho^{\prime}}$ mit

$$
\frac{\varrho}{\cos \vartheta}=\frac{\varrho^{\prime}}{\cos \vartheta^{\prime}}
$$

Zum Beweise legen wir das Koordinatensystem so, dass $P$ in den Ursprung, $t$ in die $x$-Achse und $\Pi$ in die $x, y$-Ebene fällt. Es sei $P_{n}=\left(x_{n}, y_{n}, z_{n}\right)$ und $P_{n}^{\prime}=\left(x_{n}^{\prime}, y_{n}^{\prime}, z_{n}^{\prime}\right)$; ferner seien $\vartheta_{n}$ und $\vartheta_{n}^{\prime}$ die Winkel der $z$-Achse gegen die Ebenen durch $t$ und $P_{n}$ bzw. $P_{n}^{\prime}$, so dass

$$
z_{n}=y_{n} \operatorname{tg} \vartheta_{n}, \quad z_{n}^{\prime}=y_{n}^{\prime} \operatorname{tg} \vartheta_{n}^{\prime}
$$

Da die Winkel zwischen $\boldsymbol{P}_{n} \boldsymbol{P}_{n}^{\prime}$ und der $x$-Achse nach unten beschränkt sind, kann der Richtungsfaktor

$$
\frac{x_{n}-x_{n}^{\prime}}{y_{n}-y_{n}^{\prime}}
$$

nur endliche Häufungswerte haben. Wegen

$$
\frac{y_{n}}{x_{n}} \rightarrow \mathrm{o}, \quad \frac{y_{n}^{\prime}}{x_{n}^{\prime}} \rightarrow \mathrm{o}
$$

folgt hieraus

$$
\lim \frac{x_{n}^{\prime}}{x_{n}}=\mathbf{I}
$$

Nach (4) erhält man ferner, weil die $x, y$-Ebene Tangentialebene im scharfen Sinne ist,

$$
\lim \frac{z_{n}-z_{n}^{\prime}}{y_{n}-y_{n}^{\prime}}=\lim \frac{z_{n}-z_{n}^{\prime}}{\frac{z_{n}}{\operatorname{tg} \vartheta_{n}}-\frac{z_{n}^{\prime}}{\operatorname{tg} \vartheta_{n}^{\prime}}}=0
$$


so dass auch

$$
\lim \frac{z_{n}^{\prime}}{z_{n}}=\mathbf{I}
$$

ist. Nun haben die Tangenten- und Normalenabschnitte (vgl. $\S$ I, S. 5) der beiden Punktfolgen folgende Werte:

$$
\boldsymbol{\tau}_{n}=x_{n}, \quad \sigma_{n}=\frac{z_{n}}{\cos \vartheta_{n}} ; \quad \tau_{n}^{\prime}=x_{n}^{\prime}, \quad \sigma_{n}^{\prime}=\frac{z_{n}^{\prime}}{\cos \vartheta_{n}^{\prime}}
$$

Nach Voraussetzung ist also

$$
\lim \frac{x_{n}^{2} \cos \vartheta_{n}}{2 z_{n}}=\varrho
$$

aus (5) und (6) folgt daher die Behauptung:

$$
\lim \frac{\boldsymbol{\tau}_{n}^{\prime 2}}{2 \sigma_{n}^{\prime}}=\lim \frac{x_{n}^{\prime 2} \cos \vartheta_{n}^{\prime}}{2 z_{n}^{\prime}}=\frac{\varrho \cos \vartheta^{\prime}}{\cos \vartheta}
$$

Für Kurven auf Flächen bedeutet der Satz folgendes: ${ }^{16}$

Die Fläche $\Phi$ habe in $P$ eine Tangentialebene im scharfen Sinne. $x$ und $x^{\prime}$ seien zwei von $P$ ausgehende Kurven auf $\Phi$ mit derselben Halbtangente $t$ in $P$ und Schmiegebenen, die mit der Flächennormalen in $P$ die Winkel $\vartheta \neq \frac{\pi}{2}$ und $\vartheta^{\prime} \neq \frac{\pi}{2}$ bilden. Dann gilt sowohl für die oberen als auch für die unteren Krü̈mmungen von $x$ und $x^{\prime}$ in $P$

$$
\frac{\varrho}{\cos \vartheta}=\frac{\varrho^{\prime}}{\cos \vartheta^{\prime}}
$$

Es sei nämlich $\left\{P_{n}\right\}$ eine beliebige gegen $P$ konvergierende Punktfolge auf x mit dem Krümmungsradius $\varrho ; P_{n}^{\prime}$ sei ein Punkt von $x^{\prime}$, der in derselben zu $t$ senkrechten Ebene wie $P_{n}$ liegt. Dann hat nach dem soeben bewiesenen Satze $\left\{P_{n}^{\prime}\right\}$ die Krümmung $\frac{\mathrm{I}}{\varrho^{\prime}}$ mit (3); aus der Willkürlichkeit der Folge $\left\{P_{n}\right\}$ folgt die Behauptung.

${ }^{16}$ Der folgende Satz ist im Wesentlichen mit dem von HJELmșLev (a. a. O.) äquivalent, welcher eine stetige Tangentialebene in der Umgebung von $P$ voraussetat. Eine Bemerkung von Bovligand (Géométrie infinitésimale directe, Paris I932, S. I73-I74) über die Notwendigkeit von weiteren, wesentlich schärferen Voraussetzungen könnte insofern missverständlich sein, als in seinem Beispiel die partiellen Ableitungen unstetig sind. 
Für eine spätere Anwendung geben wir noch eine Folgerung aus dem Meusnierschen Satze an. Wenn zwei Flächen $\Phi_{1}$ und $\Phi_{2}$ in einem gemeinsamen Punkte $P$ nicht zusammenfallende Tangentialebenen im scharfen Sinne haben, so gibt es in einer Umgebung von $P$ noch weitere Punkte, die beiden Flächen gemeinsam sind. Diese Schnittpunkte bilden eine Kurve $x$, die in $P$ eine Tangente $t$ im scharfen Sinne besitzt. Haben nun die Normalschnitte von $\boldsymbol{\Phi}_{1}$ und $\boldsymbol{\Phi}_{2}$ in der Richtung $t$ in $P$ endliche positive Krïmmungsradien $\varrho_{1}$ und $\varrho_{9}$, so hat $x$ in $P$ eine Schmiegebene (und daher auch eine Krümmung).

Es sei nämlich $\left\{P_{n}\right\}$ eine beliebige gegen $P$ konvergierende Punktfolge auf $x$, mit einem Krümmungsradius $\varrho$ und einer Schmiegebene, die mit den Flächennormalen in $P$ die Winkel $\vartheta_{1}$ und $\vartheta_{2}$ bildet. Dann ist

$$
\frac{\varrho}{\cos \vartheta_{i}}=\varrho_{i}
$$

Nun ist entweder $\vartheta_{1}+\vartheta_{2}$ oder $\left|\vartheta_{1} \rightarrow \vartheta_{2}\right|$ einer der Winkel zwischen den beiden Flächennormalen. Bezeichnen wir diesen mit $\nu$, so ist $\nu \neq 0$, und aus $(7)$ erhält man in beiden Fällen

$$
\cos v+\sin v \operatorname{tg} \vartheta_{1}=\frac{\varrho_{1}}{\varrho_{2}}
$$

Für $\vartheta_{1}$ kommen also nur zwei Werte in Frage, woraus man schliesst, dass $x$ in $P$ eine Schmiegebene besitzen muss. Denn wenn es auf $x$ zwei Punktfolgen $P_{n} \rightarrow P$ und $P_{n}^{\prime} \rightarrow P$ mit verschiedenen Schmiegebenen $\Sigma$ und $\Sigma^{\prime}$ gäbe, so wäre jede Ebene eines Winkelraums zwischen $\Sigma$ und $\Sigma^{\prime}$ Schmiegebene einer bestimmten Punktfolge auf $x$.

Die Lage der Schmiegebene ergibt sich aus den jeweiligen Grössenverhältnissen. In dem Falle, den wir später brauchen werden, ist

$$
\frac{\varrho_{1}}{\varrho_{2}}<|\cos \nu|
$$

Da $\sin \nu$ und $\operatorname{tg} \vartheta_{1}$ positiv sind, kann hier die Schmiegebene den spitzen Winkel zwischen den Flächennormalen nicht zerlegen.

Es mag hier noch eine bekannte Beziehung Erwähnung finden, die bereits unter den hier gemachten Voraussetzungen richtig ist. Die Fläche $\Phi$ habe in $P$ die Tangentialebene $I$ im scharfen Sinne, und der Normalschnitt mit der Tangente $t$ 
habe in $P$ die endliche Krümmung $\frac{\mathrm{I}}{\varrho_{v}}$. Fine Flächenkurve $x$ mit der Tangente $t$ in $P$ hat in $P$ dann und nur dann eine endliche Krïmmung I/Q, wenn ihre Projektion auf $I I$ eine Krümmung $\frac{\mathrm{I}}{\varrho_{g}}$ hat; dann gilt

$$
\frac{\mathrm{I}}{\varrho^{2}}=\frac{\mathrm{I}}{\varrho_{g}^{2}}+\frac{\mathrm{I}}{\varrho_{v}^{2}}
$$

Zum Beweise machen wir $P$ zum Ursprung, $t$ zur $x$-Achse und $I I$ zur $x, y$-Ebene. $\boldsymbol{\Phi}$ habe die Darstellung $z=f(x, y)$; die Projektion $x^{\prime}$ sei durch $y=g(x)$ dargestellt. Es ist

$$
\frac{\mathrm{I}}{\varrho_{v}}=\lim _{x \rightarrow 0} \frac{2|f(x, \mathrm{o})|}{x^{2}},
$$

und im Falle der Existenz

$$
\begin{aligned}
& \frac{\mathrm{I}}{\varrho_{g}}=\lim \frac{2|g(x)|}{x^{2}}, \\
& \frac{\mathrm{I}}{\varrho}=\lim \frac{2 \sqrt{g^{2}(x)+f^{2}(x, g(x))}}{x^{2}} .
\end{aligned}
$$

Weil die $x, y$-Ebene im Nullpunkt Tangentialebene im scharfen Sinne ist, gilt

$$
\lim _{\substack{x \rightarrow 0 \\ y \rightarrow 0}} \frac{f(x, y)-f(x, 0)}{y}=0,
$$

und daher ist für jede Kurve $y=g(x)$, für welche $\frac{y}{x^{2}}$ beschränkt ist,

$$
\lim _{x \rightarrow 0} \frac{f(x, y)-f(x, 0)}{x^{2}}=0 .
$$

Die Richtigkeit einer der beiden Gleichungen (I I) und (I2) zieht also die der anderen nach sich, und durch Einsetzen erhält man die Beziehung (Io).

$$
\S 3 \text {. }
$$

Die Krünmungen der Normalschnitte im einzelnen Flächenpunkt.

Das konvexe Flächenstück $\Phi$ habe in $P$ die Tangentialebene $\Pi$. Durch jede von $P$ ausgehende Halbtangente $t_{\varphi}$ an $\Phi$ legen wir die durch die Flächen- 
normale begrenzte Halbebene: der entstehende Normalbalbschnitt heisse $x_{\varphi}$. Wir nennen zur Abkürzung die obere (untere) Krümmung von $x_{\varphi}$ in $P$ die $z u t_{\varphi} g e$. hörende obere (untere) Normalkrïmmung, und bezeichnen sie mit $\frac{\mathrm{I}}{\underline{\varrho}_{\varphi}}$ bzw. mit $\frac{\mathrm{I}}{\bar{\varrho}_{\varphi}}$. Zu jedem Normalschnitt gehören also zwei obere (untere) Normalkrümmungen. Wenn sämtliche Normalschnitte Krümmungen haben, so existieren alle Normalkrümmungen, und es ist $\varrho_{\Upsilon}=\varrho_{\varphi+\pi}$.

Trägt man $\sqrt{\underline{\underline{g}}_{\varphi}}$ und $\sqrt{\bar{\varphi}_{\varphi}}$ von $P$ aus auf $t_{\varphi}$ ab, so beschreiben die Endpunkte zwei Gebilde in $\Pi$, welche die untere bzw. die obere Indikatrix heissen mögen. Wenn beide zusammenfallen, so sprechen wir von der Indikatrix von $P$. Wenn alle Normalschnitte in $P$ (zweiseitige) Krümmungen haben, so ist die Indikatrix eine Mittelpunktskurve mit $P$ als Mittelpunkt. Wir denken uns dabei naturgemäss jede Halbtangente $t_{\varphi}$ mit einem unendlich fernen Punkt versehen. Ist dann etwa $\underline{\varrho}_{\varphi_{0}}=\infty$, so nennen wir die untere Indikatrix für $\varphi=\varphi_{0}$ stetig, wenn $\lim _{\varphi \rightarrow \varphi_{0}} \varrho_{\varphi}=\infty$ ist. Etwas allgemeiner sprechen wir von Indikatrizen auch dann, wenn es in $P$ zwar keine Tangentialebene gibt, aber der Tangentialkegel ein ebenes Stück enthält. In diesem Falle sind die beiden Indikatrizen nur im betreffenden ebenen Winkelraum definiert.

Zur näheren Untersuchung der Krümmungseigenschaften auch in solchen Punkten führen wir folgende Bezeichnung ein:

$r, \varphi$ seien Polarkoordinaten in der $x, y$-Ebene. Es sei $\omega$ ein fester Winkel, $0<\omega \leq 2 \pi$, und für $0 \leq \varphi \leq \omega$ stelle

$$
z=f(r, \varphi), \quad f \geq 0
$$

ein konvexes Flächenstïck $\Phi$ dar, dessen ebene Schnitte $\varphi=$ konst. im Koordinatenursprung Tangenten haben, die sämtlich in der $x, y$-Ebene liegen. Wenn also insbesondere $\omega=2 \pi$ ist, so ist $\boldsymbol{P}$ ein innerer Punkt von $\Phi$, und die $x, y$-Ebene ist Tangentialebene von $\Phi$ in $P$. Im Folgenden denken wir uns den Winkel $\varphi$ immer auf das Intervall $o \leq \varphi \leq \omega$ beschränkt.

Die zur Richtung $\varphi$ gehörende untere bzw. obere Normalkrümmung hat den Wert

$$
\frac{\mathrm{I}}{\bar{\varrho}_{\uparrow}}=\lim _{r \rightarrow 0} \frac{2|f(r, \varphi)|}{r^{2}} \text { bzw. } \frac{\mathrm{I}}{\underline{\varrho}_{\varphi}}=\varlimsup_{r \rightarrow 0} \frac{2|f(r, \varphi)|}{r^{2}} .
$$

Es sei $s_{h}$ die Schnittkurve von $\Phi$ mit der Ebene $z=\frac{h}{2}$. Wir projizieren $s_{h}$ auf 
die $x, y$-Ebene und vergrössern die Projektion von $P$ aus ähnlich im Masstab $\sqrt{h}: \mathrm{r}$; die so entstehende Kurve heisse $\sigma_{h}$. Die Kurvengleichungen lauten:

$(2)$

$$
s_{h}: \quad f(r, \varphi)=\frac{h}{2}, \quad z=\frac{h}{2}
$$

$$
\sigma_{h}: \quad f(\sqrt{h} r, \varphi)=\frac{h}{2}, \quad z=0
$$

Wir können die Operation, welche die Kurve $s_{h}$ in $\sigma_{h}$ überführt, auch als Abbildung der Fläche auf den betrachteten Winkelraum der $x, y$-Ebene auffassen: der (auf $s_{h}$ liegende) Flächenpunkt $Q\left(r, \varphi_{0}, \frac{h}{2}\right)$ hat als Bildpunkt den (auf $\sigma_{h}$ liegenden) Punkt $Q^{\prime}\left(r^{\prime}=\frac{r}{\sqrt{h}}, \varphi_{0}, o\right)$.

Es sei nun $\left\{P_{n}\right\}$ eine gegen $P$ konvergierende Folge von Flächenpunkten die alle auf dem Normalschnitt $\varphi=\varphi_{0}$ liegen. Sind $\left(r_{n}, \varphi_{0}, \frac{h_{n}}{2}\right)$ die Koordinaten von $P_{n}$, so ist $P_{n}^{\prime}=\left(r_{n}^{\prime}=\frac{r_{n}}{\sqrt{h_{n}}}, \varphi_{0}, o\right)$ sein Bildpunkt, und es ist nach (I):

$$
\sqrt{{\underline{\underline{\rho_{p}}}}_{\rho_{0}}} \leq \underline{\lim } r_{n}^{\prime} \leq \overline{\lim } r_{n}^{\prime} \leq \sqrt{\overline{\underline{Q}}_{\varphi_{0}}} .
$$

Man sieht ohne weiteres, dass es zu jeder zwischen $\sqrt{\varrho_{\varphi_{0}}}$ and $\sqrt{\bar{\varrho}_{\varphi_{0}}}$ gelegenen Zahl $r^{\prime}$ eine solche Punktfolge $\left\{P_{n}\right\}$.gibt, dass

$$
\lim r_{n}^{\prime}=r^{\prime}
$$

wird. Betrachtet man also für eine Folge $h_{n} \rightarrow$ o den Schnittpunkt von $\sigma_{h_{n}}$ mit der Halbgeraden $\varphi=\varphi_{0}$, so erfüllen die Häufungspunkte dieser Punkte für sämtliche Folgen $h_{n} \rightarrow$ o genau ${ }^{17}$ die Strecke $\sqrt{\underline{\rho}_{\varphi_{0}}} \leq r^{\prime} \leq \sqrt{\bar{\rho}_{\varphi_{0}}}$ auf $\varphi=\varphi_{0}$. Wir erhalten auf diese Weise zwanglos die beiden Indikatrizen von $P$, zu deren bequemeren Studium wir folgende Bezeichnungen einführen:

$\gamma_{n}$ sei der Durchschnitt, $\Gamma_{n}$ die Vereinigungsmenge der durch die Kurven

${ }^{17}$ Man sieht bereits daraus, dass in $P$ dann und nur dann alle Normalschnitte einseitige Krümmungen haben, wenn die Kurven $\sigma_{h}$ für $h \rightarrow 0$ konvergieren. Die Grenzküve ist dann die Indikatrix, die daher stets eine konvexe Kurve ist. 
$\sigma_{h}$ für $h<\mathrm{I} / n$ begrenzten abgeschlossenen Bereiche. ${ }^{18}$ Für $n \rightarrow \infty$ wächst $\gamma_{n}$ monoton, während $\Gamma_{n}$ monoton fällt; wir setzen

$$
\gamma=\lim \gamma_{n}, \quad \quad \quad \Gamma=\lim \Gamma_{n}
$$

(welche Bereiche selbstredend auch unbeschränkt sein können).

\section{Die untere Indikatrix.}

Die untere Indikatrix liegt offenbar ganz auf dem Rande von $\gamma$. Andrerseits ist $\gamma_{n}$ als Durchschnitt konvexer Bereiche selbst konvex, und dasselbe gilt daher auch von $\gamma$. Der Punkt $P$ liegt im Inneren oder auf dem Rande von $\gamma$; im letzten Falle enthält der Rand von $\gamma$ eventuell eine oder zwei von $P$ ausgehende Strecken: abgesehen vom Innern dieser Strecken stimmt der Rand von $\gamma$ mit der unteren Indikatrix überein. Wir haben also:

Im Inneren jedes ebenen Stücks eines Tangentialkegels einer konvexen Fläche liegt die untere Indikatrix auf einer konvexen Kurve. Die oberen Normalkrümmungen sind daher mit Ausnahme von höchstens zwei Stellen stetig im Sinne dev Gleichung

$$
\lim _{\varphi \rightarrow \varphi_{0}} \underline{\varrho}_{\varphi}=\underline{\varrho}_{\varphi_{0}} .
$$

Die Indikatrix kann natürlich auch ganz im Unendlichen liegen. Der Punkt $P$ liegt nicht notwendig im Inneren der Indikatrix, selbst wenn $\Phi$ in $P$ eine Tangentialebene besitzt (d. h. wenn $\omega=2 \pi$ ist). Z. B. kann der Kreis $x^{2}+(y-\mathrm{I})^{2}=\mathrm{I}$ die Indikatrix darstellen; dann sind die zu den Richtungen $\pi \leq \varphi \leq 2 \pi$ gehörenden oberen Normalkrümmungen unendlich. Das ist der Fall im Punkte $u=$ o der konvexen Fläche: $x=u^{2}(\mathrm{I}+u) \cos v, y=u^{2}(\mathrm{I}+(\mathrm{I}+u) \sin v)$, $z=\frac{u v}{2} ;$ dass diese Fläche konvex ist, erkennt man z. B. daran, dass die Gausssche Krümmung in allen Punkten ausser $u=0$ positiv ist.

\section{Die obere Indikatrix.}

$\Gamma_{n}$ ist als Vereinigungsmenge konvexer, $P$ enthaltender Bereiche ein Sternbereich mit $P$ als Zentrum, d. h. wenn $Q^{\prime}$ ein Punkt von $I_{n}$ ist, so liegen alle Punkte der Strecke $\overline{P Q^{\prime}}$ in $\boldsymbol{I}_{n}$. Daher ist auch $\Gamma=\lim \Gamma_{n}$ ein Sternbereich mit $P$ als Zentrum. Die Endpunkte der von $P$ ausgehenden und $\Gamma$ bildenden Strecken

${ }^{18}$ Wenn wir vom Inneren der Kurve $\sigma_{h}$ sprechen, so meinen wir natürlich das von $\sigma_{h}$ und den Halbgeraden $\varphi=0$ und $\varphi=\omega$ begrenzte Gebiet. 
bilden offenbar die obere Indikatrix. Diese Aussage lässt sich noch verschärfen. Wenn $Q^{\prime}=\left(r^{\prime}, \varphi_{0}\right)$ ein im Endlichen gelegener Punkt von $\Gamma$ ist, so gibt es eine Folge $h_{n} \rightarrow$ o derart, dass der Schnittpunkt von $\sigma_{h_{n}}$ mit $\varphi=\varphi_{0}$ gegen $Q^{\prime}$ strebt. Aus der Folge $\left\{\sigma_{h_{n}}\right\}$ können wir eine konvergente Teilfolge auswählen (§ I, Schluss); die Grenzkurve ist natürlich konvex und enthält nach Definition $\gamma$ im Inneren. Ist also $Q^{\prime}$ ein Punkt von $\Gamma$, so ist die konvexe Hülle von $Q^{\prime}$ und der unteren Indikatrix in $\Gamma$ enthalten.

Nun mögen die oberen Normalkrümmungen in $P$ sämtlich endlich sein; dann schneidet $\gamma$ auf jedem Halbstrahl $\varphi=$ konst. eine Strecke ab, und aus dem letzten Satze folgt unmittelbar, dass für $0 \leq \varphi \leq \omega$

$$
\frac{\lim _{\varphi \rightarrow \varphi_{0}}}{\bar{\varphi}_{\varphi}} \geq \overline{\boldsymbol{\varrho}}_{\varphi_{0}}
$$

ist, d. h. dass die obere Indikatrix nach unten halbstetig ist. Für o $<\varphi<\omega$ kann aber die Ungleichung nicht statthaben, Denn zu jedem $\varphi_{1}$ mit $\circ<\varphi_{1}<\omega$ gibt es eine in $\gamma$ enthaltene Strecke $s$, die auf $\varphi=\varphi_{1}$ senkrecht steht. Die konvexe Hülle von $s$ und jedem Punkt von $\Gamma$ liegt ganz in $\Gamma$, woraus mühelos

$$
\varlimsup_{\varphi \rightarrow \varphi_{1}} \bar{\varrho}_{\varphi} \leq \bar{\varrho}_{\varphi_{1}}
$$

folgt. Wir haben somit:

Die zu den Richtungen eines ebenen Winkelraums eines Tangentialkegels von $\boldsymbol{\Phi}$ gehörenden oberen Normalkrïmmungen scien endlich; dann sind die unteren Normalkrümmungen in jeder inneren Richtung stetig im Sinne der Gleichung

$$
\lim _{\varphi \rightarrow \varphi_{0}} \bar{\varrho}_{\varphi}=\bar{\varrho}_{\varphi_{0}} .
$$

Für die beiden, den ebenen Winkelraum begrenzenden Richtungen $\varphi_{0}=0$ und $\varphi_{0}=\omega$ gilt

$$
\lim _{\varphi \rightarrow \varphi_{0}} \overline{\boldsymbol{\rho}}_{\varphi \varphi} \geq \overline{\boldsymbol{\varphi}}_{\varphi_{0}} .
$$

Ob die obere Indikatrix bei endlichen oberen Normalkrümmungen noch mehr charakteristische Eigenschaften hat, bleibt dahingestellt. ${ }^{19}$

${ }^{10}$ Zusatz bei der Korrektur. Dieses ist nicht der Fall. Es besteht nämlich, wie wir an einer anderen Stelle zeigen werden, der folgende Satz: Es sei $\gamma$ eine den Punkt $P$ im Inneren enthaltende konvexe Kurve, und $\boldsymbol{I}$ eine Kurve, die mit irgend einem Punkt $Q^{\prime}$ zugleich die konvexe Hülle von $Q^{\prime}+\gamma$ umfasst; dann gibt es eine konvexe Fläche, für die im Punkte $P$ die obere Indikatrix gerade $\Gamma$ ist, während die untere mit $\gamma$ zusammenfällt. Mit denselben Hilfsmitteln kann man auch die Unstetigkeitspunkte der Indikatrizen untersuchen, und es ergibt sich leicht, dass jede konvexe Kurve, die $P$ im Inneren oder auf dem Rande enthält, als Indikatrix auftreten kann; wir haben daher den ursprünglich hier vorgesehenen Beweis dafür gestrichen (vgl. die Bemerkung auf S. I8).

3-35150. Acta mathematica. 66. Imprimé le 26 juillet 1935. 


\section{Die Indikatrix.}

Es sei $\left\{\varphi_{n}\right\}$ eine in $o \leq \varphi \leq \omega$ dichte Folge von Richtungen, und die Normalschnitte $\varphi=\varphi_{n}$ mögen in $P$ endliche (einseitige) Krümmungen haben. Dann schneiden $\gamma$ und $\Gamma$ auf den Halbgeraden $\varphi=\varphi_{n}$ dieselben Strecken aus, und aus den eben bewiesenen Eigenschaften dieser Bereiche folgt daher, dass die beiden Indikatrizen von $P$ zusammenfallen. Wir haben also:

Fallen im Flächenpunkt $P$ für eine in $0 \leq \varphi \leq \omega$ dichte Menge von Richtungen die obere und untere Normalkrïmmung zusammen, und sind sie endlich, so existiert in $P$ eine Indikatrix. Diese ist stets eine konvexe Kurve.

Jede konvexe Kurve $x$ kann als Indikatrix eines Punktes $P$ einer konvexen Fläche auftreten, und zwar kann $P$ jeder im Inneren ${ }^{19}$ von $x$ gelegene Punkt sein. $x$ habe nämlich in ebenen Polarkoordinaten $\lambda, \varphi$ die Gleichung $\lambda=\lambda(\varphi)$. Dann hat die Fläche

$$
z=\frac{r^{2}}{2 \lambda^{2}(\varphi)}
$$

im Punkte $r=0$ eine Tangentialebene, und dieser Punkt hat $x$ als Indikatrix. Dass die Fläche konvex ist, erkennt man z. B. daran, dass die ebenen Schnitte $z=$ konst. ähnliche und ähnlich gelegene konvexe Kurven sind, während die Schnitte $\varphi=$ konst. Parabeln sind.

\section{Eine Gleichmässigkeitseigenschaft.}

Es sei $\left\{P_{n}\right\}$ eine gegen $P$ konvergierende Punktfolge auf $\Phi$ mit der Tangente $t_{\varphi_{0}}$. Wir setzen etwa $P_{n}=\left(r_{n}, \varphi_{n}, \frac{h_{n}}{2}\right)$, so dass $\varphi_{n} \rightarrow \varphi_{0}$. Der Näherungskreis des Normalhalbschnitts mit der Richtung $\varphi_{n}$ hat den Radius

$$
\varrho\left(P_{n}\right)=\frac{r_{n}^{2}+\frac{h_{n}^{2}}{4}}{h_{n}}=r_{n}^{\prime 2}+\frac{h_{n}}{4},
$$

wo $\left(r_{n}^{\prime}=\frac{r_{n}}{\sqrt{h_{n}}}, \varphi_{n}, 0\right)$ der $P_{n}$ entsprechende Punkt auf $\sigma_{h_{n}}$ ist. Nun ist offenbar, falls $\underline{\varrho}_{\varphi}$ und $\bar{\varrho}_{\varphi}$ in $\varphi_{0}$ stetig sind,

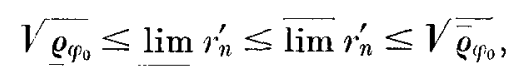

also auch

$$
\underline{\boldsymbol{Q}}_{\varphi_{0}} \leq \lim \varrho\left(\boldsymbol{P}_{n}\right) \leq \varlimsup \lim \varrho\left(\boldsymbol{P}_{n}\right) \leq \overline{\boldsymbol{\varphi}}_{\varphi_{0}} .
$$


Wenn der Punkt $P$ eine Indikatrix besitzt, so ist $\underline{\varrho}_{\varphi_{0}}=\bar{\varrho}_{\varphi_{0}}$; wir haben damit gefunden:

Die Fläche $\boldsymbol{\Phi}$ habe in $P$ eine Indikatrix, und es sei $\left\{P_{n}\right\}$ eine gegen $P$ konvergierende Punktfolge auf $\boldsymbol{\Phi}$ mit der Tangente $t_{\varphi_{0}}$; ferner sei $\varrho_{\varphi}$ in $\varphi_{0}$ stetig. Dann streben die Radien der Kreise durch $P$ und $P_{n}$, deren Ebenen senkrecht zu $I$ sind und die $\Pi$ in $P$ berühren, gegen den zur Richtung $t_{\varphi_{0}}$ gehörenden Normalkrümmungsradius. Die Radien der Näherungskreise der Normalhalbschnitte approximieren also ausserhalb beliebig kleiner Umgebungen der etwaigen (höchstens zwei) Unstetigkeitsstellen der Indikatrix die Normalkrümmungsradien gleichmässig.

\section{Konjugierte Richtungen. Hauptkrümmungsrichtungen.}

Wir wollen nun untersuchen, was von den mit dem Begriff der konjugierten Richtungen zusammenhängenden Tatsachen erhalten bleibt. Wir setzen voraus, dass die Fläche in einer Umgebung von $P$ eine Tangentialebene besitzt, und awar soll die Tangentialebene $\Pi$ in $P$ mit der Fläche nur $P$ gemeinsam haben. In $P$ selbst soll es eine Indikatrix geben, die $P$ im Inneren enthält, und nicht ganz im Unendlichen liegt (d.h. die Normalkrümmungen sollen endlich sein, und nicht sämtlich verschwinden).

Wir beweisen zunächst folgenden Satz über das durch parallele Normalen vermittelte sphärische Bild des Flächenstücks $\Phi$ :

Es sei $Q$ ein Punkt der Indikatrix von $P$, in dem diese eine Tangente $\tau$ besitzt, und $x$ eine von $P$ ausgehende Flächenkurve, die in $P$ die Tangente $P Q$ und eine endliche obere Krïmmung besitzt. Dann hat das sphärische Bild von $x$ im Bildpunkt von $P$ eine Tangente, und zwar steht diese senkrecht zu $\tau$.

Zum Beweise machen wir $\Pi$ zur $x, y$-Ebene, und $P Q$ zur positiven $x$-Achse; das Flächenstück $\Phi$ habe die Darstellung $z=f(x, y) . \quad \vec{x}$ sei zunächst der Schnitt der Fläche mit der Ebene $y=z \operatorname{tg} \vartheta$, mit $\vartheta \neq \frac{\pi}{2}$. Wir führen in der Kurvenebene rechtwinklige Koordinaten $x$ und $u=\frac{z}{\cos \vartheta}$ ein; $x$ hat dann die Gleichung

$$
u \cos \vartheta=f(x, u \sin \vartheta)
$$

Da $x$ konvex ist und nach Voraussetzung in $P$ eine rechte Kxümmung hat, existiert (vgl. \$ I, S. 7) für $x=0$ die rechte zweite Ableitung von $u$ nach $x$, und zwar ist nach dem Meusnierschen Satze 


$$
\frac{d^{2} u}{d x^{2}}=\lim _{x \rightarrow+0} \frac{-f_{x}(x, y)}{x\left[f_{y}(x, y) \sin \vartheta-\cos \vartheta\right]}=f_{x x}(0,0) \cdot \frac{\mathrm{I}}{\cos \vartheta},
$$

wobei $f_{x x}(\mathrm{o}, \mathrm{o})$ die zweite rechte Ableitung bezeichnet. Wegen $f_{y}(\mathrm{o}, \mathrm{o})=\mathrm{o}$ ist also längs jedes ebenen Schnitts durch die $x$-Achse

$$
\lim _{x \rightarrow+0} \frac{f_{x}(x, y)}{x}=f_{x x}(\mathrm{o}, \mathrm{o})
$$

Nach (2) sind die Tangenten der Kurven $s_{h}$ und $\sigma_{h}$ in entsprechenden Punkten parallel; die Tangente an $s_{h}$ hat aber den Richtungstangens

$$
-\frac{f_{x}(x, y)}{f_{y}(x, y)}
$$

Weil die Kurven $\sigma_{h}$ nach der Schlussbemerkung des $\S$ I mit ihren Tangenten gegen die Indikatrix konvergieren, ist längs $x$

$$
\lim _{x \rightarrow+0} \frac{f_{x}(x, y)}{f_{y}(x, y)}=-\operatorname{tg} \alpha
$$

wo $\alpha$ den Winkel zwischen der Tangente $\tau$ der Indikatrix und der $x$-Achse bezeichnet. Aus (3) und (4) folgt die Existenz von

$$
\lim _{x \rightarrow+0} \frac{f_{y}(x, y)}{x}=-\frac{1}{\operatorname{tg} \alpha} \cdot f_{x x}(\mathrm{o}, \mathrm{o})
$$

wobei $(x, y)$ wieder längs $x$ zu nehmen ist.

Die Koordinaten des sphärischen Bildes des Flächenpunktes $(x, y, f(x, y))$, d. h. die Richtungskosinus der Flächennormalen sind nun

$$
\frac{-f_{x}}{\sqrt{\mathrm{I}+f_{x}^{2}+f_{y}^{2}}}, \frac{-f_{y}}{\sqrt{\mathrm{I}+f_{x}^{2}+f_{y}^{2}}}, \frac{\mathrm{I}}{\sqrt{\mathrm{I}+f_{x}^{2}+f_{y}^{2}}} .
$$

Nähert man sich längs $x$ dem Nullpunkt, so besitzen diese Grössen nach (3) und (5) für $x=0$ Ableitungen nach $x$, also auch nach der Bogenlänge von $x$; diese Ableitungen sind gleich

$$
-f_{x x}(\mathrm{o}, \mathrm{o}), \quad \frac{f_{x x}(\mathrm{o}, \mathrm{o})}{\operatorname{tg} \alpha}, \quad \text { o. }
$$

Der Vektor mit diesen Komponenten steht in der Tat senkrecht auf $\tau$, womit die Behauptung für ebene Schnitte bewiesen ist. Jede Flächenkurve endlicher oberer Krümmung mit der Tangente $\underline{P Q}$ verläuft aber nach dem Meusnierschen 
Satze in einer Umgebung von $P$ zwischen zwei ebenen Schnitten mit derselben Tangente; ihr sphärisches Bild muss daher die gleiche Tangente besitzen, wie das Bild dieser ebenen Schnitte.

Von besonderem Interesse ist der Fall, dass $\underline{P Q}$ senkrecht zu $\tau$ steht; wir wollen eine solche Richtung Hauptkrümmungsrichtung nennen. Die Normalkrümmung nimmt in einer Haptkrïmmungsrichtung einen Extremwert im schwachen Sinne an. ${ }^{20}$ Für eine Hauptkrümmungsrichtung ist $\alpha=\pi / 2$, und die Grössen (6) nehmen die Werte $f_{x x}(0,0)$, o, o, an. Daher gilt folgender Spezialfall unsres Satzes:

Es sei $x$ eine von $P$ ausgehende Flächenkurve, die in $P$ eine endliche obere Krïmmung und eine Tangente besitzt, welche in eine Hauptkrïmmungsrichtung fällt. Ist dann $\mathrm{I} / \boldsymbol{r}$ die zugehörige Normalkrïmmung, und $\xi$ der Flächennormalenvektor, so gilt in $P$

$$
\frac{d \xi}{d s}=\frac{\mathrm{I}}{r} \cdot \eta
$$

wobei $s$ die Bogenlänge und $\eta$ den Einheitsvektor der Tangente von $x$ bezeichnet. Dieses ist das genaue Analogon der Formel von Olinde Rodrigues.

Wir wollen unsren Satz noch geometrisch interpretieren. Es sei $P^{\prime}$ ein variabler Punkt, und $g\left(P^{\prime}\right)$ die Schnittgerade der Tangentialebene in $P^{\prime}$ mit derjenigen in $P$. Wir lạssen $P^{\prime}$ längs einer Kurve $x$ mit der Tangente $t$ und endlicher oberer Krümmung in $P$ gegen $P$ streben: wenn dann $g\left(P^{\prime}\right)$ für jede solche Kurve derselben Grenzlage zustrebt, nennen wir diese Gerade konjugiert zu $t$. Dann können wir unsren Satz so formulieren:

Wenn die Indikatrix von $P$ im Punkte $Q$ eine Tangente $\tau$ hat, so existiert eine zu $\underline{P Q}$ konjugierte Gerade, und zwar ist sie parallel zu $\tau$. Insbesondere stehen die zu den Hauptkrümmungsrichtungen konjugierten Geraden senkrecht auf ihnen.

Der Beweis ergibt sich unmittelbar daraus, dass auf der Kugel konjugierte Richtungen senkrecht zueinander stehen, und die Tangente des sphärischen Bildes von $x$ senkrecht zu $t$ ist.

Wenn $t$ konjugiert zu $t^{\prime}$ ist, so braucht deshalb $t^{\prime}$ nicht konjugiert zu $t$ zu sein. Die Indikatrizen, für welche diese Beziehung symmetrisch ist, lassen sich alle angeben ${ }^{21}$; es sind nicht nur die Ellipsen.

${ }^{20}$ Wir haben vorausgesetzt, dass die Indikatrix. in $Q$ eine Tangente hat; da aber auch Ecken auftreten können, braucht nicht jede Richtung, in der die Normalkrümmung einen Extremwert annimmt, eine Hauptkrümmungsrichtung zu sein. Es gibt daher nicht in jedem Punkt mit Indikatrix eine Hauptkrümmungsrichtung, es kann deren aber beliebig viele geben.

21 Vgl. RADoN: Über eine besondere Art ebener konvexer Kurven, Ber. Verh. Sächs. Akad. Wiss., Leipzig, 68, (1916), S. I23. 


\section{Über einen Grenzwert von Blaschke.}

Wir wollen noch zeigen, wie sich eine von BLaschke herrührende geometrische Deutung der Gaussschen Krümmung auf Punkte mit beliebiger Indikatrix übertragen lässt.

$\Phi$ sei eine (nicht notwendig differenzierbare) konvexe Fläche mit einer Tangentialebene in $P$. In $P$ soll es ferner eine beschränkte Indikatrix geben, d. h. die Normalschnitte in $P$ sollen einseitige, von Null verschiedene Krümmungen haben. Wir machen wieder $P$ zum Ursprung und die Flächennormale zur $z$-Achse, und stellen die Fläche in Zylinderkoordinaten in der Form $z=f(r, \varphi)$ dar, etwa mit $f \geq 0$. Es handelt sich dann um eine Abschätzung der Oberfläche $\Omega(h)$ der durch die Ebene $z=h$ abgeschnittenen Flächenkappe. $r=\bar{r}(\varphi)$ sei die Gleichung der Projektion des Flächenschnitts $s_{2 h}$ mit der Ebene $z=h, \Sigma(h)$ der Inhalt dieser konvexen Kurve. Da der Flächeninhalt einer geschlossenen konvexen Fläche stets grösser ist als derjenige, einer ganz in ihr enthaltenen konvexen Fläche ${ }^{22}$, ist $\Omega(h)$ kleiner als $\Sigma(h)$ vermehrt um den Inhalt des senkrechten Zylinders mit dem Querschnitt $s_{2 h}$ zwischen $z=0$ und $z=h$. Wir haben also

$$
\Sigma(h)<\Omega(h) \leq \Sigma(h)+2 \pi h \cdot \max \bar{r}(\varphi),
$$

und daher

$$
\varlimsup_{\underline{\lim }} \frac{\Omega(h)}{h}=\varlimsup_{\underline{\lim }} \frac{\Sigma(h)}{h}
$$

Nun ist

$$
\Sigma(h)=\frac{\mathrm{I}}{2} \int_{0}^{2 \pi} \bar{r}^{2}(\varphi) d \varphi
$$

hierin strebt $\frac{\bar{r}^{2}(\varphi)}{2 h}$ gegen den zur Richtung $\varphi$ gehörenden Normalkrümmungsradius $\varrho(\varphi)$, und zwar gilt wegen der Gleichmässigkeitseigenschaft dieser Konvergenz (vgl. S. I 8-I9)

$$
\lim \frac{\Omega(h)}{h}=\lim \frac{\boldsymbol{\Sigma}(h)}{h}=\int_{0}^{2 \pi} \varrho(\varphi) \cdot d \varphi
$$

oder in Worten:

Die Fläche $\Phi$ habe in $P$ eine Tangentialebene und eine beschränkte Indikatrix. Bezeichnet $\Omega(h)$ den Flächeninhalt der Kappe, die von der zur Tangentialebene in $P$ 
parallelen Ebene im Abstand $h$ abgeschnitten wird, so existiert $\lim 2 \pi \cdot \frac{\Omega(h)}{h}$ und ist gleich dem arithmetischen Mittel der Normalkrümmungsradien in $P$.

Ist die Indikatrix insbesondere eine Ellipse, so kann man $\varrho$ in der Form

$$
\frac{I}{\varrho}=\frac{\cos ^{2} \varphi}{\varrho_{1}}+\frac{\sin ^{2} \varphi}{\varrho_{2}}
$$

darstellen und erhält

$$
\lim \frac{\Omega(h)}{h}=\int_{0}^{2 \pi} \varrho d \varphi=2 \pi \sqrt{\varrho_{1} \varrho_{2}}
$$

oder

$$
\varrho_{1} \varrho_{2}=\lim \left(\frac{\Omega(h)}{2 \pi h}\right)^{2}
$$

dieses ist die Formel von BLaschKe. ${ }^{23}$

Es sei noch bemerkt, dass die durch die sphärische Abbildung der Fläche definierte Gausssche Krümmung, falls sie existiert, bei allgemeiner Indikatrix nicht gleich dem Blaschkeschen Grenzwert ist. Bildet man nämlich die zum Punkte $r=0$ wie eben definierte Flächenkappe mit dem Inhalt $\Omega(h)$ durch parallele Normalen auf die Kugel ab, und ist $\Omega^{*}(h)$ der Inhalt des Bildes, so ist z. B. für die konvexe Fläche

$$
\begin{gathered}
z=\frac{r^{2}}{1+\sin ^{2} \varphi} \\
\lim \left(\frac{\Omega(h)}{2 \pi h}\right)^{2}=\frac{9}{16}, \quad \lim \frac{\Omega(h)}{\Omega^{*}(h)}=\frac{2 \sqrt{2}}{5}
\end{gathered}
$$

$\$ 4$.

Fast überall vorhandene Kruìmmungseigenschaften der Normalschnitte.

Wir verlassen nun die Frage nach den im einzelnen Punkt bestehenden Eigenschaften der Normalkrümmungen, und suchen mit Hilfe der Theorie der reellen Funktionen schärfere, dafür aber nur fast überall gültige Aussagen zu gewinnen.

${ }^{28}$ vgl. W. BlaschKE: Differentialgeometrie I, Berlin I924, 2. Aufl, S. 85 . 
$z=f(x, y)$ stelle ein konvexes Flächenstück $\Phi$ dar, dessen Stützebenen nirgends zur $x, y$-Ebene senkrecht stehen. Jeder ebene Schnitt. der Fläche hat als konvexe Kurve fast überall eine Tangente. Nach dem üblichen Schlussverfahren bilden daher für jedes feste $\alpha$ die Flächenpunkte, in denen der Flächenschnitt mit einer Ebene $y=x \operatorname{tg} \alpha+$ Konst. keine Tangente hat, eine Nullmenge $\Lambda_{\alpha}$. Es seien $\alpha$ und $\beta$ zwei Winkel, und $A=\Lambda_{\alpha}+\Lambda_{\beta}$. Dann haben in jedem Flächenpunkt, der nicht in $A$ liegt, zwei ebene Schnitte der Fläche eine Tangente: Die Nullmenge $A$ besteht daher genau aus denjenigen Punkten, in denen $\Phi$ keine Tangentialebene hat.

Ferner besitzen alle ebenen Flächenschnitte in fast allen Punkten auch eine endliche (zweiseitige) Krümmung. Ist also $\alpha$ ein fester Winkel, so bilden die Flächenpunkte, in denen der Flächenschnitt mit der Ebene $y=x \operatorname{tg} \alpha+$ Konst. keine endliche Krümmung hat, eine Nullmenge $\Lambda_{\alpha}^{\prime}$. Nun sei die Folge $\left\{\alpha_{n}\right\}$ dicht im Intervall $\left(-\frac{\pi}{2}, \frac{\pi}{2}\right)$, und

$$
\Lambda^{\prime}=\sum_{n} \Lambda_{\alpha_{n}}^{\prime}
$$

Natürlich ist $\Lambda<\Lambda^{\prime}$, und $\Lambda^{\prime}$ ist wieder eine Nullmenge. Wenn der Punkt $P\left(x_{0}, y_{0}\right)$ nicht in $A^{\prime}$ liegt, haben alle Schnitte der Fläche mit den Ebenen $y-y_{0}=\left(x-x_{0}\right) \operatorname{tg} \alpha_{n}$ in $P$ eine endliche Krümmung. Da anf konvexen Flächen jede Tangentialebene zugleich Tangentialebene im scharfen Sinne ist (vgl. $\$ 2$, S. 9), können wir den Meusnierschen Satz anwenden und schliessen, dass auch die zugehörigen Normalschnitte in $P$ eine endliche Krümmung besitzen. Daher hat $P$ eine Indikatrix (vgl. $\S_{3}$, S. I 8), und zwar ist diese, weil die rechten und linken Krümmungen der Normalschnitte zusammenfallen, eine Kurve mit $P$ als Mittelpunkt. $\Lambda^{\prime}$ besteht umgekehrt nur aus solchen Punkten, in denen nicht alle Normalschnitte endliche zweiseitige Krümmungen haben. Es gilt also folgender Satz:

Auf jedem konvexen Flächenstück $\Phi$ gibt es eine Nullmenge $\Lambda^{\prime}$ derart, dass in jedem $\Lambda^{\prime}$ nicht angehörendem Flächenpunkte sämtliche ebenen Schnitte endliche Krümmungen haben. Die Indikatrix ist in diesen Punkten eine konvexe Kurve mit dem Flächenpunkt als Mittelpunkt.

Es gilt aber noch mehr. Wir wollen einen Flächenpunkt $P$, in dem die Indikatrix ein Kegelschnitt (Ellipse oder Geradenpaar) mit $P$ als Mittelpunkt ist, als normalen Flächenpunkt bezeichnen. Es soll gezeigt werden, dass von einer. 
Nullmenge $A^{*}$ abgesehen, alle Flächenpunkte normal sind, d. h. dass in ihnen der Eulersche Satz gilt. ${ }^{24}$

Wir werden zunächst nicht auf der Fläche, sondern in der $x, y$-Ebene operieren. Ist $\mu$ eine Punktmenge auf der Fläche, so bezeichnen wir ihre Projektion auf die $x, y$-Ebene mit $\mu$; insbesondere ist also $\Phi$ der Definitionsbereich von $f(x, y)$. Ist $\mu$ eine Nullmenge auf $\Phi$, so ist auch $\mu$ eine Nullmenge, und umgekehrt. Das kann man z. B. daraus entnehmen, dass für konvexe Flächen in der Form $z=f(x, y)$ die bekannte Oberfiächenformel gilt. ${ }^{25}$

Nach § I, S. 7 folgt bei konvexen Kurven aus der Existenz der Krümmung die der zweiten Ableitung. In allen Punkten von $\Phi$, die nicht der Nullmenge $\underline{\Lambda}^{\prime}$ angehören, haben also die Schnitte der Fläche mit allen Ebenen $y=x \operatorname{tg} \alpha+$ Konst. als Funktion der Länge auf den Spurgeraden dieser Ebenen in der $x, y$-Ebene erste und zweite Ableitungen. Wir bezeichnen diese mit $f_{\alpha}(x, y)$ und $f_{\alpha \alpha}(x, y)$. Es ist z. B.

$$
f_{a}\left(x_{0}, y_{0}\right)=\left.\cos \alpha \cdot \frac{d}{d x} f\left(x,\left(x-x_{0}\right) \operatorname{tg} \alpha+y_{0}\right)\right|_{x=x_{0}}
$$

und insbesondere $f_{\frac{\pi}{2}}=f_{y}$. Unser erstes Ziel ist eine Beziehung zwischen den Ableitungen $f_{\alpha \alpha}\left(x_{0}, y_{0}\right)$ als Funktion von $\alpha$ in einem festen Punkt $P$ abzuleiten.

Dazu führen wir die zu $f(x, y)$ gehörige additive Intervallfunktion $F(I)$ ein: Ist $I$ ein durch die Ungleichungen

$$
x_{0}-h \leq x<x_{0}+h, \quad y_{0}-k \leq y<y_{0}+k
$$

definiertes Intervall, das in $\Phi$ liegt, so ordnen wir ihm als Funktionswert ${ }^{26}$ die Zahl

$$
\begin{aligned}
F(I)=\Delta\left(x_{0}, y_{0} ; h, k\right)=f\left(x_{0}+h, y_{0}+k\right) & +f\left(x_{0}-h, y_{0}-k\right)- \\
& -f\left(x_{0}-h, y_{0}+k\right)-f\left(x_{0}+h, y_{0}-k\right)
\end{aligned}
$$

24 Es sei bemerkt, dass der folgende Beweis die Konvexität der Fläche nur schwach ausnutzt. Der Beweis gilt für jede Fläche $z=f(x, y)$, wenn nur i) $f$ von beschränkter Schwankung ist, und 2) in fast allen Punkten $\left(x_{0}, y_{0}\right)$ die Funktion $f\left(x, y_{0}+\left(x-x_{0}\right) \operatorname{tg} \alpha\right)$ für jedes $\alpha$ eine zweite Ableitung besitzt.

${ }^{25}$ vgl. S. SAKs, Théorie de l'Intégrale, Warszawa 1933, S. I 2 I (Satz von Tonelli).

20 Bei hinreichend regulärem $f(x, y)$ ist natürlich

$$
F(I)=\int_{x_{0}-h}^{x_{0}+h} \int_{y_{0}-k}^{y_{0}+k} f x y(x, y) d x d y
$$

4-35150. Acta mathematica. 66. Imprimé le 26 juillet 1935. 
zu. Diese Funktion ist absolut additiv: Wenn $I=\sum I_{n}$ eine Zerlegung von $I$ in endlich oder abzählbar viele Teilintervalle ist, so gilt, da $f(x, y)$ als konvexe Funktion von beschränkter Schwankung ist,

$$
F(I)=\sum F\left(I_{n}\right)
$$

Die Funktion $F(I)$ kann daher in fast allen Punkten nach Intervallen differenziert werden, worunter man folgendes versteht: Es seien $h_{n} \rightarrow$ o und $k_{n} \rightarrow 0$ zwei positive Nullfolgen mit

$$
\frac{\mathrm{I}}{N} \leq \frac{h_{n}}{k_{n}} \leq N
$$

wobei $N \geq$ I beliebig vorgegeben ist; dann existiert für alle Punkte $\left(x_{0}, y_{0}\right)$, die nicht in einer bestimmten Nullmenge $\Lambda_{N}^{1}$ liegen, der Grenzwert

$$
\lim _{n \rightarrow \infty} \frac{\Delta\left(x_{0}, y_{0} ; h_{n}, k_{n}\right)}{4 h_{n} k_{n}},
$$

und ist unabhängig von der speziellen Wahl der Folgen $\left\{h_{n}\right\}$ und $\left\{k_{n}\right\} .{ }^{27}$ Die Menge $\underline{\Lambda}_{N}^{1}$ wächst monoton mit $N$, und ihre Grenzmenge ist wieder eine Nullmenge, die wir mit $\underline{\Lambda}^{1}$ bezeichnen. Für jeden Punkt $\left(x_{0}, y_{0}\right)$, der $\underline{A}^{1}$ nicht angehört, und jedes Paar positiver Nullfolgen $\left\{h_{n}\right\}$ und $\left\{k_{n}\right\}$ mit

$$
0<\underline{\lim } \frac{h_{n}}{k_{n}} \leq \varlimsup \lim \frac{h_{n}}{k_{n}}<\infty
$$

existiert

$$
\lim \frac{A\left(x_{0}, y_{0} ; h_{n}, k_{n}\right)}{4 h_{n} k_{n}}=a\left(x_{0}, y_{0}\right)
$$

Bei hinreichend regulärem $f(x, y)$ ist $a\left(x_{0}, y_{0}\right)=f_{x y}\left(x_{0}, y_{0}\right)$.

Aus (2) erhält man die gesuchte Beziehung zwischen den $f_{\alpha \alpha}\left(x_{0}, y_{0}\right)$. Es sei $\left(x_{0}, y_{0}\right)$ ein Punkt, der weder $\underline{\Lambda}^{\prime}$ noch $\underline{\Lambda}^{1}$ angehört. Wir setzen in (2) $k_{n}=$ $=h_{n} \operatorname{tg} \alpha$, und erhalten so für jedes $o \leq \alpha<\frac{\pi}{2}$

${ }^{27}$ Einen Beweis dieses bekannten Satzes der Lebesgueschen Theorie findet man z. B. bei S. SAKs, a. a. O. S. 49 . Dort wird allerdings $h_{n}=k_{n}$ vorausgesetzt, d. h. es wird nur nach Quadraten differenziert. Indessen überträgt sich der Beweis wörtlich auf unsere Voraussetzungen, da es nur auf die Gültigkeit des Vitalischen Satzes ankommt. 
(3)

$$
\begin{aligned}
a\left(x_{0}, y_{0}\right)=\lim _{h \rightarrow 0} \frac{\mathrm{I}}{4 h^{2} \operatorname{tg} \alpha}\left[f\left(x_{0}+h, y_{0}+h \operatorname{tg} \alpha\right)+f\left(x_{0}-h, y_{0}-h \operatorname{tg} \alpha\right)-\right. \\
\\
\left.-f\left(x_{0}+h, y_{0}-h \operatorname{tg} \alpha\right)-f\left(x_{0}-h ; y_{0}+h \operatorname{tg} \alpha\right)\right] .
\end{aligned}
$$

Da $\left(x_{0}, y_{0}\right)$ nicht in $\underline{A}^{\prime}$ liegt, existieren $f_{\alpha \alpha}\left(x_{0}, y_{0}\right)$ und $f_{-\alpha,-\alpha}\left(x_{0}, y_{0}\right)$. Nach $\S$ I (7) gilt

$$
\begin{array}{r}
f_{\alpha \alpha}=\cos ^{2} \alpha \cdot \lim _{h \rightarrow 0} \frac{f\left(x_{0}+h, y_{0}+h \operatorname{tg} \alpha\right)+f\left(x_{0}-h, y_{0}-h \operatorname{tg} \alpha\right)-2 f\left(x_{0}, y_{0}\right)}{h^{2}}, \\
f_{-\alpha,-\alpha}=\cos ^{2} \alpha \cdot \lim _{h \rightarrow 0} \frac{f\left(x_{0}+h, y_{0}-h \operatorname{tg} \alpha\right)+f\left(x_{0}-h, y_{0}+h \operatorname{tg} \alpha\right)-2 f\left(x_{0}, y_{0}\right)}{h^{2}} .
\end{array}
$$

Setzt man das in (3) ein, so erhält man

$$
f_{c \alpha}\left(x_{0}, y_{0}\right)-f_{-\alpha,-\alpha}\left(x_{0}, y_{0}\right)=4 \sin \alpha \cos \alpha a\left(x_{0}, y_{0}\right) .
$$

Das ist die gewünschte Beziehung.

Wir drehen nun das Koordinatensystem $x, y$ um einen Winkel $\omega$ mit irrationalem Verhältnis $\frac{\omega}{\pi}$. Das neue System heisse $\xi, \eta$, und $f(x, y)$ gehe dabei in $\varphi(\xi, \eta)$ über. Wie eben gehört zu $\varphi(\xi, \eta)$ eine Nullmenge $\Lambda^{2}+\Lambda^{\prime}$ ausserhalb deren die Ableitung $\varphi_{\beta \beta}$ für jedes $\beta$ existiert und der Beziehung

$$
\varphi_{\beta \beta}\left(\xi_{0}, \eta_{0}\right)-\varphi_{-\beta,-\beta}\left(\xi_{0}, \eta_{0}\right)=4 \sin \beta \cos \beta \cdot b
$$

genügt. Hierin ist der Winkel $\beta$ natürlich von der $\xi$-Achse an gerechnet; führt man statt $\beta$ den Winkel $\alpha$ mit der $x$-Achse ein und schreibt (5) in den alten Koordinaten, so erhält man die Beziehung

$$
f_{\alpha \alpha}\left(x_{0}, y_{0}\right)-f_{2 \omega-\alpha, 2 \omega-\alpha}\left(x_{0}, y_{0}\right)=4 \sin (\alpha-\omega) \cos (\alpha-\omega) \cdot b\left(x_{0}, y_{0}\right) .
$$

Die beiden Beziehungen (4) und (6) gelten gleichzeitig in jedem Punkte $\left(x_{0}, y_{0}\right)$, der nicht der Nullmenge $\underline{\Lambda}^{*}=\underline{\Lambda}^{1}+\underline{\Lambda}^{2}+\Lambda^{\prime}$ angehört. Wir wollen daraus schliessen, dass $f_{\alpha \alpha}\left(x_{0}, y_{0}\right)$ eine quadratische Form in $\cos \alpha$ und $\sin \alpha$ ist.

$f_{\alpha \alpha}\left(x_{0}, y_{0}\right)$ ist nach $\S 3$, S. I 8 eine stetige Funktion von $\alpha$. Wir zeigen nun, dass die beiden Funktionalgleichungen

$$
\begin{aligned}
& \text { a) } g(\alpha)-g(-\alpha)=2 a \sin 2 \alpha \\
& \text { b) } g(\alpha)-g(2 \omega-\alpha)=2 b \sin 2(\alpha-\omega)
\end{aligned}
$$


bei gegebenem $a, b$ und $g(0)$ die Funktion

$$
g(\alpha)=A \sin 2 \alpha+A^{\prime} \cos 2 \alpha+A^{\prime \prime}
$$

mit

$$
A=a, \quad A^{\prime}=\frac{a \cos \omega-b}{\sin 2 \omega}, \quad A^{\prime \prime}=g(0)-A^{\prime}
$$

und nur diese Funktion als stetige Lösung haben. (8) ist offenbar eine quadratische Funktion in $\cos \alpha$ und $\sin \alpha$. Man verifiziert leicht, dass (8) die Gleichungen (7) löst. Andrerseits sind durch $(7)$ die Funktionswerte $g(2 n \omega), n=\mathrm{I}, 2, \ldots$, bestimmt. Aus ( 7 b) folgt nämlich

$$
g(2 \omega)=g(0)+2 b \sin 2 \omega
$$

wenn nun $g(2 n \omega)$ bestimmt ist, so bekommt man aus $(7 \mathrm{a})$ auch $g(-2 n \omega)$ und dann aus $(7 \mathrm{~b})$ für $\alpha=-2 n \omega$

$$
g(2(n+\mathrm{I}) \omega)=g(-2 n \omega)+2 b \sin 2(2 n+\mathrm{I}) \omega .
$$

Die Winkel $2 n \omega$ liegen wegen der Irrationalität von $\frac{\omega}{\pi}$ überall dicht, daher kann es nur eine stetige Lösung von ( 7 ) geben.

Von der Nullmenge $A^{*}$ abgesehen gilt also in allen Punkten $\left(x_{0}, y_{0}\right)$ eine Beziehung der Form

$$
f_{\alpha \alpha}\left(x_{0}, y_{0}\right)=A_{1} \cos ^{2} \alpha+A_{2} \sin ^{2} \alpha+A_{3} \sin \alpha \cos \alpha
$$

wobei die Koeffizienten nur von $\left(x_{0}, y_{0}\right)$ abhängen.

Hieraus können wir die Gültigkeit des Eulerschen Satzes in allen Flächenpunkten nachweisen, die nicht über einem Punkt der Menge $\Lambda^{*}$ liegen. Es sei $P=\left(x_{0}, y_{0}, f\left(x_{0}, y_{0}\right)\right)$ ein solcher Punkt. Der Einfachheit halber denken wir uns das Koordinatensystem so gedreht, dass die $x$-Achse parallel zur Schnittgeraden der Tangentialebene in $P$ mit der $x, y$-Ebene wird. ${ }^{28}$ Das dürfen wir tun, da $f_{\alpha \alpha}$ der Definition nach invariant gegenüber Drehungen des Koordinatensystems $x, y$ in seiner Ebene ist, und die Beziehung (9) daher erhalten bleibt. Es ist dann

$$
f_{x}\left(x_{0}, y_{0}\right)=\mathrm{o} \text { und } f_{\alpha}\left(x_{0}, y_{0}\right)=f_{y}\left(x_{0}, y_{0}\right) \sin \alpha
$$

Die Argumente $x_{0}, y_{0}$ lassen wir von nun an fort. 
Die Krümmung des Flächenschnitts mit der Ebene $y-y_{0}=\left(x-x_{0}\right) \operatorname{tg} \alpha$ in $P$ ist

$$
\frac{\mathrm{I}}{\varrho_{\alpha}}=\frac{f_{\alpha \alpha}}{\sqrt{\mathrm{I}+f_{\alpha}^{2}}}=\frac{f_{\alpha \alpha}}{\sqrt{\mathrm{I}+f_{y}^{2} \sin ^{2} \alpha}} .
$$

$t_{\alpha}$ sei die Tangente dieses Schnitts in $P, \varphi=\varphi(\alpha)$ der Winkel zwischen $t_{\alpha}$ und $t_{0}$, schliesslich $\nu$ der Neigungswinkel der Tangentialebene in $P$.gegen die $x, y$-Ebene. Dann ist

$$
\begin{aligned}
& \cos \nu=\frac{\mathrm{I}}{\sqrt{\mathrm{I}+\bar{f}_{y}^{2}}} \\
& \operatorname{tg} \alpha=\cos \nu \operatorname{tg} \varphi
\end{aligned}
$$

und danach

$$
\begin{aligned}
& \sin \varphi=\frac{\sin \alpha}{\sqrt{\mathrm{I}+f_{y}^{2} \sin ^{2} \alpha}} \cdot \sqrt{\mathrm{I}+f_{y}^{2}} \\
& \cos \varphi=\frac{\cos \alpha}{\sqrt{\mathrm{I}+f_{y}^{2} \sin ^{2} \alpha}}
\end{aligned}
$$

Für den Winkel $\vartheta=\mathfrak{g}(\alpha)$ zwischen der Kurvenebene und der Flächennormale findet man

$$
\cos \vartheta=\sqrt{\frac{\mathrm{I}+\overline{f_{y}^{2} \cdot \sin ^{2} \alpha}}{\mathrm{I}+f_{y}^{2}}}
$$

Unter Benutzung des Meusnierschen Satzes ergibt sich aus (10) und (12) für die Krümmung $\frac{I}{r}$ des Normalschnitts mit der Tangente $t_{\alpha}$

$$
\frac{\mathrm{I}}{r}=\frac{\cos \vartheta}{\varrho}=\frac{\mathrm{I}}{\sqrt{\mathrm{I}+f_{y}^{\prime \prime}}} \cdot \frac{f_{c \alpha}}{\mathrm{I}+f_{y}^{2} \sin ^{2} \alpha} .
$$

Aus (9), (I I) und (I3) folgt

$$
\frac{\mathrm{I}}{r}=a_{1} \cos ^{2} \varphi+a_{2} \sin ^{2} \varphi+a_{3} \sin \varphi \cos \varphi
$$

wo die Koeffizienten nur vom Punkte $P$ abhängen. (I4) ist der Eulersche Satz, der besagt, dass die Indikatrix ein Kegelschnitt ist. Da sie bei uns konvex sein muss, kommt nur die Ellipse und das Geradenpaar in Betracht. Wir haben damit bewiesen: 
Fast alle Punkte eines konvexen Flächenstücks sind normal, d.h. von einer Nullmenge abgesehen ist die Indikatrix in jedem Flächenpunkt $P$ eine Ellipse oder ein Geradenpaar mit $P$ als Mittelpunkt.

Zusammenfassend können wir sagen: Auf $\boldsymbol{\Phi}$ gibt es drei Nullmengen $\Lambda<\Lambda^{\prime}<\Lambda^{*}$ derart, dass es in allen Punkten von $\Phi-\Lambda$ Tangentialebenen und in allen Punkten von $\Phi-\Lambda^{\prime}$ endliche Normalkrïmmungen in allen Richtungen gibt. Schliesslich gilt in den Punkten von $\boldsymbol{\Phi}-\Lambda^{*}$ sogar der Eulersche Satz. Analytisch sind diese drei Mengen folgendermassen charakterisiert: $\Phi-\Lambda$ besteht aus den Punkten, in denen $f_{\alpha}$ für jedes a existiert; $\Phi-\Lambda^{\prime}$ aus denjenigen, in denen auch $f_{\alpha \alpha}$ existiert. Nun führen wir in der $x, y$-Ebene auf alle mögliche Weisen rechtwinklige Koordinatensysteme ein. $\Phi-\Lambda^{*}$ besteht dann aus denjenigen Punkten der $x, y$-Ebene, in denen die Fläche in allen diesen Systemen nach ähnlichen achsenparallelen Rechtecken differenzierbar ist, d. h. wenn $\left(x_{0}, y_{0}\right)$ ein Punkt von $\Phi-\Lambda^{*}$ ist, so existiert in jedem dieser Koordinatensysteme ${ }^{29}$ für jedes $\alpha$ (vgl. (I)):

$$
\lim _{h \rightarrow 0} \frac{\Delta\left(x_{0}, y_{0} ; h, h \operatorname{tg} \alpha\right)}{4 h^{2} \operatorname{tg} \alpha},
$$

und ist unabhängig von $\alpha$.

$$
\S 5 .
$$

\section{Über den Nabelpunktsatz.}

Beim Beweise des Satzes, dass eine aus lauter Nabelpunkten ${ }^{30}$ bestehende Fläche ein Kugelstück ist, wird gewöhnlich mindestens dreimalige Differenzierbarkeit vorausgesetzt. Die einmalige Differenzierbarkeit ist trivialerweise unentbehrlich. Dagegen sind die anderen Voraussetzungen unnötig. Es gibt aber geschlossene differenzierbare konvexe Flächen, auf denen fast alle Punkte Nabelpunkte sogar derselben Krümmung sind, und die trotzdem keine Kugeln sind (vgl. \$ 6). Es liegt daher nahe zu fragen, unter welchen Regularitätsannahmen es zur Charakterisierung der Kugel genügt zu wissen, dass fast alle Punkte Nabelpunkte sind.

${ }^{29}$ Unser Beweis zeigt nämlich, dass hierfür in einem festen Koordinatensystem die Beziehung (4) notwendig und hinreichend ist. Wenn aber (4) in zwei Koordinatensystemen erfüllt ist, so ist, wie wir sahen, $f_{\alpha \alpha}$ eine quadratische Form in $\cos \alpha$ und $\sin \alpha$, so dass die Beziehung (4) dann in allen Koordinatensystemen gilt.

${ }^{80}$ Unter einem Nabelpunkt verstehen wir einen Punkt, dessen Indikatrix ein konzentrischer Kreis ist, oder ganz im Unendlichen liegt. 
Wir schicken folgenden Hilfsatz voraus: $z=f(x, y)$ stelle ein differenzierbares konvexes Flächenstïck $\Phi$ dar, dessen Stützebenen nirgends zur $x, y$-Ebene senkrecht stehen. $k$ sei ein ebener Schnitt von $\Phi$, welcher in einem Punkte $P$ Normalschnitt ist. Schliesslich seien $f_{x}(x, y)$ und $f_{y}(x, y)$ längs $k$ (etwa als Funktionen der Länge) totalstetig ${ }^{14}$, und fast alle Punkte von $k$ seien Nabelpunkte der Fläche. Dann ist $k$ in allen seinen Punkten Normalschnitt der Fläche.

Beweis. Nach § 3, (7) fällt die Ableitung der Flächennormale in allen Punkten von $k$, die Nabelpunkte sind, in die Tangente von $k$. Der Winkel $\omega$, den die Flächennormale längs $k$ mit der Ebene von $k$ bildet, hat also fast überall auf $k$ eine verschwindende Ableitung. Wegen der Totalstetigkeit von $f_{x}$ und $f_{y}$ ist dieser Winkel selbst totalstetig, und daher ist er konstant. In $P$ ist $\omega=0$, so dass durchweg $\omega=0$ ist.

Sind insbesondere alle Punkte von $k$ Nabelpunkte der Fläche, so verschwindet die Ableitung von $\omega$ in allen Punkten von $k$, woraus $\omega=0$ folgt, ohne dass man die Totalstetigkeit von $f_{x}$ und $f_{y}$ besonders voraussetzen muss. Wenn also $\Phi$ aus lauter Nabelpunkten besteht, so ist jeder ebene Schnitt, der in einem seiner Punkte Normalschnitt ist, auch in allen seinen Punkten Normalschnitt der Fläche, woraus man mühelos schliesst, dass $\Phi$ ein Kugelstück ist Daher:

Eine aus lauter Nabelpunkten positiver Krïmmung bestehende differenzierbare Fläche ist ein Kugelstück. ${ }^{31}$

Aus unsrem Hilfsatz kann man den Nabelpunktsatz noch in verschiedenen Fassungen ableiten, die aber alle in der folgenden enthalten sind: Durch zwei Punkte $P$ und $P^{\prime}$ von $\boldsymbol{\Phi}$ gebe es je eine auf $\Phi$ dichte Menge von Normalschnitten, auf denen fast alle Punkte Nabelpunkte der Fläche und die Funktionen $f_{x}$ und $f_{y}$ totalstetig sind. Dann ist $\Phi$ ein Kugelstück. Nach dem Hilfsatz sind nämlich die genannten Normalschnitte durch $P$ und $P^{\prime}$ in allen ihren Punkten Normalschnitte der Fläche. Wegen der Stetigkeit der Flächennormale gilt dasselbe von allen Normalschnitten durch $P$ und $P^{\prime}$. Nun sieht man leicht, dass eine differenzierbare Fläche, auf der alle Normalschnitte durch einen Punkt $P$ durchweg

${ }^{81}$ Die Konvexität braucht nicht besonders vorausgesetzt zu werden, da die Tangentialebene in einem solchen Nabelpunkt zugleich Stützebene im Kleinen ist. Eine Fläche, die in jedem Punkt eine Stützebene im Kleinen besitzt, ist aber konvex. Einen auf E. ScHмIDT zurückgehenden Beweis des entsprechenden Satzes für ebene Kurven findet man bei L. Bieberbach: Differentialgeometrie, Berlin I932, S. $20 \mathrm{f}$. Der Beweis überträgt sich unmittelbar auf $n$ Dimensionen (vgl. B.-F. S. 6-7). 
Normalschnitte sind, eine Rotationsfläche mit $P$ als $P o l$ ist. Weil vorausgesetzt wurde, dass die Tangentialebene nirgends $\operatorname{zur} x, y$-Ebene senkrecht steht, können $P$ und $P^{\prime}$ nicht Gegenpunkte sein, so dass $\Phi$ auf zwei verschiedene Weisen als Rotationsfläche aufgefasst werden kann; daraus folgt die Behauptung unmittelbar.

Ein konvexes Flächenstück $z=f(x, y)$ ist demnuch sicher ein Kugelstück wenn: I) fast alle Punkte Nabelpunkte sind, und 2) $f_{x}(x, y)$ und $f_{y}(x, y)$ totalstetig als Funktionen beider Veränderlichen sind. Diese Bedingungen sind viel natürlicher als die ersten, welche wir nur deshalb angegeben haben, weil sie zeigen, dass man an sich nur von einer Nullmenge von Punkten zu wissen braucht, dass sie Nabelpunkte sind.

In einem normalen Flächenpunkt ist die Indikatrix eine Ellipse oder ein Geradenpaar; gehen also von einem normalen Punkt drei Richtungen aus, welche zu je zweien einen kleineren Winkel als $\pi$ bilden und in denen die Normalkrümmungen gleich sind, so ist die Indikatrix ein Kreis. Andrerseits wissen wir, dass fast alle Punkte einer konvexen Fläche normal sind. Die Bedingung I) ist demnach sicher erfüllt, wenn von fast jedem Flächenpunkt drei Tangentialrichtungen ausgehen, von denen keine zwei entgegengesetzt sind, und deren zugehörige untere oder oberen Normalkrïmmungen gleich sind.

Der folgende Paragraph wird zeigen, dass die zweite Voraussetzung nicht fortgelassen werden darf, selbst wenn man verlangt, dass alle Nabelpunkte dieselbe Krümmung haben. Wir wollen nun noch beweisen, dass $f_{x}(x, y)$ und $f_{y}(x, y)$ als Funktionen beider Veränderlichen totalstetig sind, wenn die oberen Normalkrümmungen der Fläche beschränkt sind.

Betrachten wir nämlich ein Flächenstück, auf dem $f_{x}$ und $f_{y}$ beschränkt sind, so folgt nach dem Meusnierschen Satze aus der Beschränktheit der oberen Normalkrümmungen die Beschränktheit der oberen Krümmungen der Schnitte der Fläche mit allen Vertikalebenen $a x+b y+c=0$. Daher sind auch die oberen Derivierten $\bar{f}_{\alpha \alpha}$ gleichmässig beschränkt (vgl. $\$ 4$, (Io)), d. h. es ist

$$
\left|\bar{f}_{\alpha \alpha}(x, y)\right|<M
$$

Für $\alpha=0$ und $\alpha=\pi / 2$ ergeben sich hieraus die beiden Lipschitzbedingungen

$$
\left|f_{x}\left(x_{1}, y\right)-f_{x}\left(x_{0}, y\right)\right|<M\left|x_{1}-x_{0}\right|
$$

$$
\left|f_{y}\left(x, y_{1}\right)-f_{y}\left(x, y_{0}\right)\right|<M\left|y_{1}-y_{0}\right|
$$


Für $\alpha=\frac{\pi}{4}$ schliesst man ferner (vgl. \& 4, S. 27)

$$
\frac{\mathrm{I}}{4 h^{2}}|f(x+h, y+h)+f(x-h, y-h)-f(x-h, y+h)-f(x+h, y-h)|<2 \sqrt{2} M .
$$

Da man jedes Rechteck in fremde. seitenparallele Quadrate zerlegen kann, ist auch

$$
\frac{\mathrm{I}}{4 h k}|f(x+h, y+k)+f(x-h, y-k)-f(x-h, y+k)-f(x+h, y-k)|<2 \sqrt{2} M
$$

Daher gilt für die oberen Derivierten $\bar{f}_{x y}$ und $\bar{f}_{y x}$ ebenfalls

$$
\left|\bar{f}_{x y}\right| \leq 2 \sqrt{2} M,\left|\bar{f}_{y x}\right| \leq 2 \sqrt{2} M
$$

Damit haben wir die weiteren Lipschitzbedingungen

$$
\begin{aligned}
& \left|f_{x}\left(x, y_{1}\right)-f_{x}\left(x, y_{0}\right)\right| \leq 2 \sqrt{2} M\left|y_{1}-y_{0}\right| \\
& \left|f_{y}\left(x_{1}, y\right)-f_{y}\left(x_{0}, y\right)\right| \leq{ }_{2} \sqrt{2} M\left|x_{1}-x_{0}\right|
\end{aligned}
$$

Aus (2) und (3) folgt die behauptete Totalstetigkeit von $f_{x}$ und $f_{y}{ }^{32}$

Wir geben noch eine Anwendung dieser Beziehungen. A us (2) und (3) folgt, dass auch die Komponenten der Flächennormalen

$$
\frac{f_{x}}{\sqrt{\mathrm{I}+f_{x}^{2}+f_{y}^{2}}}, \quad \frac{f_{y}}{\sqrt{\mathrm{I}+f_{x}^{2}+f_{y}^{2}}}, \quad \frac{-\mathrm{I}}{\sqrt{\mathrm{I}+f_{x}^{2 y}+f_{y}^{\prime \prime}}}
$$

Lipschitzbedingungen in beiden Veränderlichen erfüllen. Diese Grössen liefern aber eine Darstellung des sphärischen Bildes der Fläche mit den Parametern $x$ und $y$. Aus dem Erfülltsein der Lipschitzbedingungen folgt ${ }^{33}$ die Richtigkeit der bekannten Formel für die Oberfläche: Ist $B$ ein Bereich der $x, y$-Fibene und $S(B)$ der Flächeninhalt des sphärischen Bildes von $B$, so gilt

$$
S(B)=\iint_{B} \frac{f_{x x} f_{y y}-f_{x y}^{2}}{\sqrt{\mathrm{I}+f_{x}^{2}+f_{y}^{2}}} d x d y
$$

(wobei der Integrand nur auf einer Nullmenge unbestimmt ist). Für die Oberfläche $O(B)$ des über $B$ liegenden Flächenstücks gilt

32 vgl. Carathéodory, Vorlesungen über reelle Funktionen, I. Aufl., Leipzig 1918, § 568.

38 T. RADó: Ủber das Flächenmass rektifizierbarer Flächen, Math. Ann. Ioo (I928).

5-35150. Acta mathematica. 66. Imprimé le 28 juillet 1935. 


$$
\dot{O}(B)=\iint_{B} \sqrt{\mathrm{I}+f_{x}^{2}+f_{y}^{2}} d x d y .
$$

Da die Integranden in (4) und (5) beschränkt sind, kann man die Integrale nach ähnlichen Rechtecken differenzieren. Für fast alle Punkte $(x, y)$ des Definitionsbereichs von $f(x, y)$ gilt also, wenn $\left\{I_{n}\right\}$ eine Folge von ähnlichen konzentrischen Intervallen ist, die sich auf $(x, y)$ zusammenzieht,

$$
\lim _{n \rightarrow \infty} \frac{S\left(I_{n}\right)}{O\left(I_{n}\right)}=\frac{f_{x x} f_{y y}-f_{x y}^{2}}{\left(\mathrm{I}+f_{x}^{2}+f_{y}^{2}\right)^{2}}
$$

Es sei nun $(x, y)$ insbesondere ein normaler Punkt. Indem man dann ein solches Koordinatensystem einführt, in dem $f_{x y}$ verschwindet, sieht man sofort, dass der Ausdruck auf der rechten Seite von (6) gerade das Produkt der Hauptkrümmungen ist. Somit:

Ist $\Phi$ eine differenzierbare konvexe Fläche mit beschränkten oberen Normalkrümmungen, so existiert die Gaussche Krümmung in fast allen normalen Punkten und ist gleich dem Produkt der Hauptkrümmungen. Das ïber die normalen Punkte von $\Phi$ erstreckte Integral der Gausschen Krümmung liefert den Flächeninhalt des sphärischen Bildes von $\boldsymbol{\Phi}$.

Unter welchen allgemeineren Voraussetzungen dieser Satz gilt, ist ungeklärt; insbesondere fragt es sich, ob die Totalstetigkeit der sphärischen Abbildung hierzu genügt. Der nächste Paragraph zeigt, dass diese Abbildung auch bei differenzierbaren konvexen Flächen nicht totalstetig zu sein braucht, und dass der Integrand in (4) für ein geschlossenes, in der Einheitskugel gelegenes $\Phi$ fast überall verschwinden kann.

$\S 6$.

Beispiel einer Fläche, die bis auf eine Nullmenge aus lauter Kugelstïcken desselben Radius besteht.

Es soll jetzt eine Fläche $\Phi$ mit folgenden Eigenschaften konstruiert werden: $\Phi$ ist eine differenzierbare, konvexe geschlossene Fläche, die ganz in der Einheitskugel liegt. Auf $\Phi$ gibt es ein Gebiet $\Gamma$, mit $^{34}$

${ }^{34}$ Wir bezeichnen mit Betragstrichen stets den Flächeninhalt bzw. das Mass der auf der Fläche gelegenen Mengen. 


$$
|I|=|\Phi|
$$

(d. h. $\Phi-\Gamma$ ist eine Nullmenge). $\Gamma$ ist Vereinigungsmenge von Gebieten, deren jedes einem Gebiet der Kugel mit dem Radius $r$ kongruent ist, wobei $r>\mathrm{I}$ beliebig vorgegeben ist; insbesondere kann auch $r=\infty$ genommen werden.

Der Flächeninhalt des sphärischen Bildes von $T$ ist offenbar höchstens gleich $\frac{|\Gamma|}{r^{2}}$, also, weil ja $\Phi$ in der Einheitskugel liegt, höchstens $\frac{4 \pi}{r^{2}}$. Daher ist das Mass des Bildes von $\Phi-T$ positiv. Das Beispiel zeigt demnach zweierlei:

I) Die sphärische Abbildung einer stetig differenzierbaren konvexen Fläche braucht nicht totalstetig zu sein; es kann fast die ganze Fläche auf eine Nullmenge der. Kugel abgebildet werden, und eine Nullmenge der Fläche auf den Rest der Kugel.

2) Es gibt konvexe, differenzierbare und in der Einheitskugel gelegene Flächen, deren Punkte bis auf eine Nullmenge sämtlich Nabelpunkte derselben Krümmung $\mathrm{I} / r<\mathrm{I}$ sind.

Unsere Konstruktion besteht in der Übertragung der bekannten Konstruktion einer monotonen, stetigen, aber nicht totalstetigen Funktion einer Veränderlichen ${ }^{35}$ auf die Kugel. Wir bringen die Einheitskugel zum Schnitt mit einer Kugel $\Sigma$ vom Radius $r$, und erhalten so eine geschlossene konvexe Fläche $\Phi^{\prime}$, die aus je einem Stück der beiden Kugeln besteht. $\Phi^{\prime}$ hat eine Kante, die wir so abrunden, dass eine differenzierbare Fläche $\Phi_{1}$ entsteht, die noch ein Stück $\Gamma_{1}$ der Kugel $\Sigma$ enthält. Nun ersetzen wir ein Stück von $\Phi_{1}-\Gamma_{1}$ durch ein Kugelstück, runden die Kante wieder ab, usf. Indem wir das Verfahren fortsetzen, erhalten wir eine monoton abnehmende Flächenfolge $\left\{\Phi_{n}\right\}$, deren Grenzfläche die gewünsehte Eigenschaft hat. Bei der Durchführung hat man auf zweierlei zu achten: I) dass sich der Flächeninhalt der Vereinigungsmenge der Kugelstücke mit dem Radius $r$, die in $\Phi_{n}$ enthalten sind, $\left|\Phi_{n}\right|$ beliebig nähert; 2) dass die Normalkrümmungen in $\Phi_{n}-I_{n}$ grösser sind als I $r$, damit man Stücke von $\Phi_{n}-\Gamma_{n}$ durch Kugelstücke vom Radius $r$ ersetzen kann. Der letzte Umstand fällt fort, wenn $r=\infty$ ist, $d . h$. wenn man die Flächenstücke durch Ebenenstiucke ersetzt. Dieser Fall wird daher etwas einfacher.

Wir schicken der Durchführung einige Hilfsbetrachtungen voraus.

Es sei $\Phi$ eine geschlossene, differenzierbare konvexe Fläche mit beschränkten oberen Normalkrümmungen, $A$ ein offenes analytisches Flächenstück auf ihr, und

35 vgl. etwa CARAThéodory, a. a. O. $\$ \S 336 / 7$. 
sämtliche Normalkrümmungen auf $A$ seien grösser als $\mathrm{I} / r$. Wenn $P$ ein Punkt von $A$ ist, so sei für jedes positive $\varepsilon \Sigma=\Sigma(P, \varepsilon)$ die Kugel vom Radius $r$, deren Mittelpunkt auf der inneren Normalen von $P$ im Abstand $r+\varepsilon$ von $P$ liegt. Der Schnitt von $\mathcal{A}$ und $\Sigma(P, \varepsilon)$ enthält bei hinreichend kleinem $\varepsilon$ eine geschlossene, in einer beliebig kleinen Umgebung von $P$ verlaufende Kurve $x=x(P, \varepsilon)$. Den von ihr auf $A$ begrenzten abgeschlossenen Bereich bezeichnen wir mit $\lambda=\lambda(P, \varepsilon)$, den kleineren auf $\Sigma$ mit $\Lambda=\Lambda(P, \varepsilon)$. Offenbar gilt; sobald nur $\varepsilon$ genügend klein ist,

$$
\frac{\left|A^{\prime}(P, \varepsilon)\right|}{|\lambda(P, \varepsilon)|}>\frac{1}{2}
$$

Wir ersetzen nun den Bereich $\lambda(P, \varepsilon)$ von $A$ durch $A(P, \varepsilon)$. Dadurch entsteht eine neue geschlossene konvexe Fläche $\Phi^{\prime}$, die in $\Phi$ enthalten ist, und auf der die Kurve $x$ eine Kante ist. Diese Kante wollen wir abrunden.

Hilfsatz 1. Bei hinreichend kleinem $\varepsilon$ gibt es eine geschlossene differenzierbare konvexe Fläche $\Phi_{1}$, die mit $\Phi^{\prime}$ ausserhalb einer vorgegebenen Umgebung von $\varkappa(P, \varepsilon)$ übereinstimmt. Der Teil von $\Phi_{1}$, der nicht auch auf $\Phi^{\prime}$ liegt, ist eine analytische Kanalfläche, deren Normalkrümmungen sämtlich beschränkt und grösser als $\mathrm{I} / \mathrm{r}$ sind.

Beweis. Es sei $\delta$ so klein gewählt, dass sämtliche Normalkrümmungen von $\Phi^{\prime}$ kleiner als $\mathrm{I} / \delta$ sind. Wir bezeichnen die inneren Parallelfächen im Abstand $\delta$ ron $\Delta$ und $\Sigma$ mit $A_{\delta}$ und $\Sigma_{\delta}$. Beide Flächen sind wieder konvex und analytisch. Ihre Schnittkurve heisse $\varkappa^{\prime}$. Wir betrachten die Einhüllende der Kugeln mit dem Radius $\delta$ und den Mittelpunkten auf $x^{\prime}$ : diese Kanalfäche berïhrt $\sigma^{\prime}$ längs zweier geschlossener Kurven; die eine verläuft im Inneren von $\Lambda(P, \varepsilon)$, die andere auf $\Delta$ im Ausseren von $\lambda(P, \varepsilon)$, so dass diese beiden Berührungskurven auf $\Phi^{\prime}$ einen Streifen um $x$ abgrenzen. Wir ersetzen nun diesen Streifen durch den von denselben Kurven begrenzten Streifen auf der Kanalfläche, und erhalten so eine geschlossene, differenzierbare, konvexe Fläche $\Phi_{1}$. (Die Konvexität folgt z. B. so: Das Innere von $\chi^{\prime}$ auf der Parallelfläche $\Sigma_{\delta}$, und das Äussere auf $\Delta_{\delta}$ bilden zusammen wieder ein konvexes Flächenstück, und man erhält $\Phi_{1}$ indem man zu dieser die äussere Parallelfäche im Abstand $\delta$ konstruiert.) $\Phi^{\prime}$ und $\Phi_{1}$ stimmen ansserhalb des erwähnten Streifens um $x$ überein, und dieser kann mit $\delta$ beliebig klein gemacht werden. Wir haben nur noch die Normalkrümmungen der Kanalfäche zu untersuchen. 
Jeder Punkt $Q$ der Kanalfläche liegt auf einem Kreis $\gamma$ vom Radius $\delta$ mit dem Mittelpunkt $Q^{\prime}$ auf $x^{\prime}$ (nämlich der Charakteristik der eingehüllten Kugelschar). Die Berührungspunkte von $\gamma$ mit $A$ und $\Sigma$ seien $D$ und $S$. Die Hauptkrümmungsrichtungen in $Q$ fallen in die Tangente von $\gamma$ und senkrecht dazu. Die erste Hauptkrïmmung ist daher $\frac{\mathrm{I}}{\delta}>\frac{\mathrm{I}}{r}$; die andere ändert sich auf $\gamma$ monoton längs jedes Viertelkreises, der durch die Haupt- und Binormale von $x^{\prime}$ in $Q^{\prime}$ aus $\gamma$ ausgeschnitten wird. Nun ist der Winkel zwischen den Flächennormalen von $A$ und $\Sigma$ bei kleinem $\varepsilon$ ebenfalls klein. Daher kann der Winkel der Flächennormalen von $\Delta_{\varepsilon}$ und $\Sigma_{\varepsilon}$ in $Q^{\prime}$ mit $\varepsilon$ beliebig klein gemacht werden. Wir wählen $\varepsilon$ so klein, dass dieser Winkel der Ungleichung $\$ 2$ (9) genügt. Dann kann die Hauptnormale von $x^{\prime}$ den Winkel der äusseren Flächennormalen in $Q^{\prime}$ nicht zerlegen ( $\S_{2}$ S. I 2 ), und daraus folgt dann, dass die Punkte $D$ und $S$ auf einem der erwähnten Viertelkreise liegen, so dass die zweite Hauptkrümmung zwischen $D$ und $S$ monoton variiert. In den Berührungspunkten $D$ und $S$ sind aber sämtliche Normalkrümmungen der Kanalläche mindestens gleich den entsprechenden der berührenden Flächen (weil sie ganz im Inneren derselben verläuft), also in $S$ mindestens gleich $\mathrm{I} / r$, und in $D$ grösser als $\mathrm{I} / r$. - Dáss die Normalkrümmungen des eingesetzten Stücks der Kanalfäche beschränkt sind, folgt z. B. daraus, dass jeder Punkt der Fläche auf einer ganz in dieser enthaltenen Kugel von Radius $\delta$ liegt.

Hilfsatz 2. Für die Bereiche $\lambda(P, \varepsilon)$ gilt der Vitalische U̇berdeckungssatz, d. h. ist $\varepsilon(P)$ irgend eine positive Funktion, so gibt es zu jedem vorgegebenem $\alpha<\mathrm{I}$ und $\delta>0$ auf $\Lambda$ endlich viele paarweise fremde Bereiche $\lambda\left(P_{v}, \varepsilon_{v}\right)$ mit $\varepsilon_{\mu}<\varepsilon\left(P_{\mu}\right)$, deren Durchmesser kleiner sind als $\delta$, und für die

$$
\sum_{v}\left|\lambda\left(P_{v}, \varepsilon_{v}\right)\right|>\alpha|\Delta|
$$

ist.

Beweis. Für die geodätischen Kreisscheiben $\beta(P, \varepsilon)$ auf $\Delta$ (d. h. für die Menge aller Punkte von $\mathcal{A}$, die von $P$ höchstens den Abstand $\varepsilon$ haben), gilt der Vitalische Satz. ${ }^{36}$ Ist nun $\beta\left(P, \varepsilon^{*}\right)$ die kleinste geodätische Kreisscheibe, die $\lambda(P, \varepsilon)$ enthält, so gilt, wie man leicht sieht,

$$
\lim _{\varepsilon \rightarrow 0} \frac{\left|\beta\left(P, \varepsilon^{*}\right)\right|}{|\lambda(P, \varepsilon)|}<a(P),
$$

${ }^{36}$ Den Beweis führt man ganz wie üblich, vgl. etwa CARAтhéodory, a. a. O. $\$ 288$. 
wo a nur von dem Verhältnis der beiden Hauptkrümmungsradien in $P$ abhängt. Daher sind die Bereiche $\beta(P, \varepsilon)$ bezüglich der Kreisscheiben regulär, und es gilt für sie der Vitalische Satz. ${ }^{37}$

Nach diesen Vorbereitungen konstruieren wir die Fläche. Wir zeigen: Es gibt eine Folge konvexer Flächen $\left\{\Phi_{n}\right\}$ mit folgenden Eigenschaften:

1) $\Phi_{n}$ ist ganz in dem von $\Phi_{n-1}$ begrenzten abgeschlossenen Bereich enthalten.

2) Auf $\Phi_{n}$ gibt es ein Gebiet $\Gamma_{n}$, das aus Kugelstücken vom festen Radius $r>$ I besteht, so dass $\Gamma_{n-1}$ in $\Gamma_{n}$ enthalten ist.

3) $\frac{\left|\Gamma_{n}\right|}{\left|\Phi_{n}\right|}$ wächst monoton gegen $\mathbf{I}$.

4) $\Phi_{n}$ zerfällt durch endlich viele rektifizierbare Kurven in analytische Flächenstücke.

5) Die Normalkrümmungen in den inneren Punkten von $\Phi_{n}-\Gamma_{n}$ sind sämtlich grösser als $\mathrm{I} / r$ und nach oben beschränkt.

Beweis. $\Phi_{1}$ sei die Einheitskugel, und $\Phi_{1}, \ldots, \Phi_{n}$ seien bereits definiert. $\Delta$ sei die Vereinigungsmenge der analytischen Gebiete, die in $\Phi_{n}-\Gamma_{n}$ enthalten sind. Nach Hilfsatz 2. können wir zu jedem $\varepsilon(P)>0$ in $\Delta$ endlich viele paarweise fremde Bereiche $\lambda\left(P_{v}, \varepsilon_{v}\right)$ so wählen, dass $\varepsilon_{\mu}<\varepsilon\left(P_{\mu}\right)$ und

$$
\sum\left|\lambda\left(P_{v}, \varepsilon_{v}\right)\right|>\frac{2}{3}|A|=\frac{2}{3}\left|\Phi_{n}-\Gamma_{n}\right|
$$

wird, und dass die Durchmesser so klein sind, wie es der im Hilfsatz I. beschriebene Abrundungsprozess erfordert. Wir wählen dabei $\varepsilon(P)$ so klein, dass diese Bereiche der Ungleichung $(*)$ genügen. Die Bereiche $\lambda\left(P_{v}, \varepsilon_{v}\right)$ sind abgeschlossen und haben daher zu je zweien einen positiven Abstand. Wir können daher den Abrundungsprozess für jeden einzelnen. dieser Bereiche so durchführen, dass die anderen dadurch nicht berührt werden. Die Zahl $\delta$ wählen wir dabei so klein, dass der nach der Abrundung vom Kugelstück $\Lambda\left(P_{p}, \varepsilon_{v}\right)$ übrig blejbende Teil $\Lambda^{\prime}\left(P_{v}, \varepsilon_{v}\right)$ der Ungleichung

$$
\left|\Lambda^{\prime}\left(P_{v}, \varepsilon_{v}\right)\right| \geq \frac{3}{4}\left|\Lambda\left(P_{y}, \varepsilon_{v}\right)\right|
$$

genügt. Die so entstehende Fläche nennen wir $\Phi_{n+1}$. Die Eigenschaft I) ist

37 Vgl. etwa CARathéodory, a. a. O. \$ 289 . 
trivialerweise erfüllt. 2) $\boldsymbol{\Phi}_{n+1}$ enthält $\Gamma_{n}$ und ausserdem noch die Vereinigungsmenge $\Gamma^{\prime}$ der Kugelstücke $\Lambda^{\prime}\left(P_{v}, \varepsilon_{v}\right)$. Wir setzen $\Gamma_{n+1}=\Gamma_{n}+\Gamma^{\prime}$. 3) Es ist

$$
\left|\Gamma^{\prime}\right|=\sum\left|\Lambda^{\prime}\left(P_{v}, \varepsilon_{v}\right)\right| \geq \frac{3}{4} \sum\left|\Lambda\left(P_{v}, \varepsilon_{v}\right)\right| .
$$

Nun liegt $\Lambda\left(P_{v}, \varepsilon_{v}\right)$ auf der inneren Seite von $\lambda\left(P_{v}, \varepsilon_{v}\right)$; daher ist (vgl. S. 22, Fussnote 22) $\left|\Lambda\left(P_{v}, \varepsilon_{v}\right)\right| \leq\left|\lambda\left(P_{v}, \varepsilon_{v}\right)\right|$. Setzen wir also

$$
\frac{\Sigma\left|\Lambda\left(P_{v}, \varepsilon_{v}\right)\right|}{\Sigma\left|\lambda\left(P_{v}, \varepsilon_{v}\right)\right|}=a_{n}
$$

so ist wegen $(*) \frac{\mathrm{I}}{2}<a_{n} \leq \mathrm{I}$, und

$$
\left|\Gamma^{\prime}\right| \geq \frac{3}{4} a_{n} \sum\left|\lambda\left(P_{v}, \varepsilon_{v}\right)\right| \geq \frac{a_{n}}{2}\left|\Phi_{n}-\Gamma_{n}\right|
$$

Somit ist

$$
\frac{\left|\Gamma_{n+1}\right|}{\left|\Phi_{n+1}\right|} \geq \frac{\left|\Gamma_{n+1}\right|}{\left|\Phi_{n}\right|}=\frac{\left|\Gamma_{n}\right|}{\left|\Phi_{n}\right|}+\frac{\left|\Gamma^{\prime}\right|}{\left|\Phi_{n}\right|} \geq \frac{\left|\Gamma_{n}\right|}{\left|\Phi_{n}\right|}+\frac{a_{n}}{2}\left(\mathrm{I}-\frac{\left|\Gamma_{n}\right|}{\left|\Phi_{n}\right|}\right)
$$

$\mid \frac{\Gamma_{n} \mid}{\left|\Phi_{n}\right|}$ wächst also monoton, und der Grenzwert ist offenbar I, Die beiden letzten Eigenschaften folgen daraus, dass $\Phi_{n+1}$ nur aus Teilen von $\Phi_{n}$, Kugelstücken und aus hinzugekommenen Streifen analytischer Kanalfächen besteht. Die Normalkrümmungen der letzteren erfüllen die Forderung.

Die Flächenfolge $\left\{\Phi_{n}\right\}$ konvergiert, etwa gegen $\Phi$. Setzen wir $\Gamma=\lim \Gamma_{n}$ so ist $\Gamma$ in $\Phi$ enthalten, und wegen $\left|\Phi_{n}-\Gamma_{n}\right| \rightarrow 0$ und $\left|\Gamma_{n}\right|<\left|\Gamma_{n+1}\right|$ ist $|\Gamma|=|\Phi|$. Wir haben also nur noch zu zeigen, dass $\Phi$ differenzierbar ist. Durch jeden Punkt $P$ von $\Phi$ gibt es unendlich viele ebene Schnitte, die bis auf eine (lineare) Nullmenge aus Kreisbögen bestehen. $\Phi$ hat in $P$ sicher eine Tangentialebene, wenn zwei solche ebene Schnitte in $P$ differenzierbar sind. Wir wollen zeigen, dass jeder solche Schnitt $\pi$ in allen seinen Punkten differenzierbar ist. Dazu genügt es zu sehen, dass die Richtungen der Tangenten an diese Kreisbögen dicht in der Menge aller Richtungen der Ebene von $\pi$ liegen. Wir versehen $\pi$ mit einem Umlaufssinn; $A_{1}$ und $A_{2}$ seien zwei Punkte von $\pi$, die auf je einem Kreisbogen $\gamma_{1}$ und $\gamma_{2}$ von $\pi$ liegen. Wir haben zu zeigen, dass es zwischen $A_{1}$ und $A_{2}$ einen weiteren Punkt gibt, der ebenfalls auf einem Kreisbogen von $x$ liegt, und dessen Tängente der Richtung nach zwischen denjenigen 
von $A_{1}$ und $A_{2}$ liegt. Wenn $\gamma_{1}=\gamma_{2}$ so ist nichts zu beweisen. Sonst haben aber $\gamma_{1}$ und $\gamma_{2}$ einen positiven Abstand voneinander, und da die Kreisbögen auf $\pi$ dicht liegen, gibt es einen weiteren Kreisbogen $\gamma$ zwischen ihnen. $\gamma_{1}, \gamma_{2}$ und $\gamma$ liegen für ein passendes $n$ bereits in $\Gamma_{n}$ also auf $\Phi_{n}$. Da diese Fläche differenzierbar ist, liegen alle Tangenten an $\gamma$ zwischen denjenigen an $\gamma_{1}$ und an $\gamma_{2}$.

\section{$\S 7$.}

\section{Kürzeste Linien.}

Die Ausführungen dieses Abschnitts sind wieder lokaler Natur, und im U̇brigen unabhängig von den $\$ \S 3-6$.

Wir betrachten die Gesamtheit der auf einer konvexen Fläche verlaufenden rektifizierbaren Linien zwischen zwei Flächenpunkten $P$ und $Q$. Dann gibt es nach Lebesgue ${ }^{7}$ wenigstens einen ron $P$ nach $Q$ führenden rektifizierbaren Bogen $g$, dessen Länge gleich ist der unteren Grenze der Längen aller dieser Kurven. $g$ heisse eine kürzeste Linie. Dass diese Bezeichnung auf $P$ und $Q$ keinen Bezug nimmt, ist dadurch gerechtfertigt, dass jeder Teilbogen von $g$ wieder eine kürzeste Linie ist. Wir untersuchen die Differenzierbarkeitseigenschaften der kürzesten Linien in solchen Flächenpunkten, in denen die Fläche eine Tangentialebene besitzt. Das Ergebnis formulieren wir vorweg:

Die konvexe Fläche $\Phi$ besitze im Punkte $P$ eine Tangentialebene II. Dann hat jede durch $P$ gehende (von $P$ ausgehende) kiurzeste Linie auf $\Phi$ in $P$ eine Tangente (Halbtangente), und ferner eine Schmiegebene, die auf $\Pi$ senkrecht steht.

Nach \$ I (4) folgt aus der Lage der Schmiegebene, dass die Projektion der kürzesten Linie auf $\Pi$ in $P$ die Krümmung Null hat.

Wir können daher den Satz auch so formulieren: Auf einer konvexen Fläche haben die kürzesten Linien in jedem Flächenpunkt mit Tangentialebene die geodätische Krïnmung Null. Das gilt auch in solchen Punkten, in denen es keine Normalkrümmungen gibt, oder diese unendlich sind.

Für den Beweis ${ }^{38}$ benötigen wir einige Hilfsbetrachtungen.

38 Kin einfaeber Ansatz seheint zunächst die Differenzierbarkeit der kürzesten Linien zu liefern. Die (nicht notwendig konvexe) Fläche $\Phi$ habe in $P$ eine Tangentialebene im scharfen Sinne, und $g$ sei eine von $P$ ausgehende kürzeste Linie. Ferner seien $\left\{Q_{n}\right\}$ und $\left\{R_{n}\right\}$ zwei gegen $P$ konvergierende Punktfolgen auf $g$, wobei etwa $Q_{n}$ zwischen $P$ und $R_{n}$ liegen möge. Bezeichnet man dann mit $g(A, B)$ die auf $g$ gemessene Entfernnng, während (wie in $\S \mathrm{I}) e(A, B)$ die Euklidische Entfernung angibt, so ist 
I. $\Phi$ sei eine geschlossene konvexe Fläche. Als Fusspunkt auf $\Phi$ eines Punktes $P$ bezeichnen wir den eindeutig definierten Punkt $F_{P}$ von $\Phi$ mit kürzester Entfernung von $P$. Der Fusspunkt hängt stetig von $P$ ab.

Hilfsatz 1. Durchläuft $P$ eine rektifizierbare Kurve $x$ im Äusseren von $\Phi$, so durchläuft der Fusspunkt $F_{p}$ von $P$ auf $\Phi$ eine rektifizierbare Kurve auf $\Phi$, deren Länge höchstens so gross ist, wie die von $x$.

Beweis. Es genügt zu zeigen: Sind $A$ und $B$ irgend zwei Punkte im Äusseren von $\Phi$, so gilt für die Entfernungen

$$
e\left(F_{A}, F_{B}\right) \leq e(A, B)
$$

$\Phi$ hat in $F_{A}$ und $F_{B}$ Stützebenen $\Pi_{A}$ und $\Pi_{B}$, die senkrecht zu den Loten $\underline{A F_{A}}$ und $\underline{B F_{B}}$ stehen (vgl. Fig. I). Wenn diese Ebenen parallel sind, so ist nichts zu beweisen. Sonst sei $h$ ihre Schnittgerade; $h_{A}$ und $h_{F_{A}}$ bezeichnen die Parallelen zu $h$ durch $A$ bzw. $F_{A}$. Die Normalebene zu $h$ durch $B$ schneide $h$ in $D$, ferner $h_{F_{A}}$ in $A_{1}$ und $h_{A}$ in $A^{\prime}$. Dann ist

$$
\begin{gathered}
e^{2}(A, B)=e^{2}\left(A, A^{\prime}\right)+e^{2}\left(A^{\prime}, B\right) \\
e^{2}\left(F_{A}, F_{B}\right)=e^{2}\left(F_{A}, A_{1}\right)+e^{2}\left(A_{1}, F_{B}\right)=e^{2}\left(A, A^{\prime}\right)+e^{2}\left(A_{1}, F_{B}\right) \\
\mathrm{I}=\frac{g\left(P, Q_{\nu}\right)+g\left(Q_{\nu}, R_{v}\right)}{g\left(P, R_{\nu}\right)} \geq \frac{e\left(P, Q_{\nu}\right)+e\left(Q_{\nu}, R_{v}\right)}{g\left(P, R_{\nu}\right)}
\end{gathered}
$$

Nun sieht man leicht (z. B. ans dem Kriterium im $\S 2$, S. 8 f.), dass

und daher auch

$$
\lim \frac{g\left(P, \boldsymbol{R}_{v}\right)}{e\left(P, \boldsymbol{R}_{\nu}\right)}=\mathrm{I}
$$

$$
\lim \frac{e\left(P, Q_{v}\right)+e\left(Q_{v}, R_{v}\right)}{e\left(P, R_{v}\right)}=\mathrm{I}
$$

ist. Wenn nun die beiden Punktfolgen einer Beziehung der Form

$$
\frac{\mathrm{I}}{a} \leq \frac{e\left(P, Q_{v}\right)}{e\left(P, R_{v}\right)} \leq a
$$

genügen, so folgt hieraus, dass der Winkel zwischen den Sehnen $\overline{P Q} n$ und $\overline{P R}_{n}$ gegen Null strebt, so dass, wenn eine der beiden Punktfolgen eine Tangente hat, die andere dieselbe Gerade als Tangente besitzt.

Indessen folgt daraus nicht die Fixistenz einer Tangente. Sind $r, \varphi$ ebene Polarkoordinaten, so ist die Kurve $\varphi=\sin \sqrt{|\log r|} \pm \frac{\pi}{2}$ rektifizierbar; sie hat im Ursprung $P$ offenbar keine Tangente. Trotzdem gilt die eben festgestellte Beziehung für je zwei Punktfolgen auf ihr, die einer Bedingung der Form $(*)$ genügen.

6-35150. Acta mathematica. 66. Imprimé le 28 juillet 1935. 
Nun möge die Parallele zu $A_{1} F_{B}$ durch $B$ die Strecke $\overline{A_{1} A^{\prime}}$ etwa in $A_{2}$ schneiden (andernfalls betrachten wir die Parallele zu $\underline{A_{1} F_{B}}$ durch $A^{\prime}$ ). Dann ist

$$
e\left(A_{2}, B\right) \geq e\left(A_{1}, F_{B}\right)
$$

da der Fusspunkt des Lotes von $B$ auf $A^{\prime} A_{1}$ auf der Verlängerung von $\overline{A^{\prime}} \overline{A_{2}}$ über $A_{2}$ hinaus liegt, ist

$$
e\left(A_{2}, B\right) \leq e\left(A^{\prime}, B\right)
$$

Setzt man dies in (4), so erhält man wegen (2) und (3) die Behauptung (I).

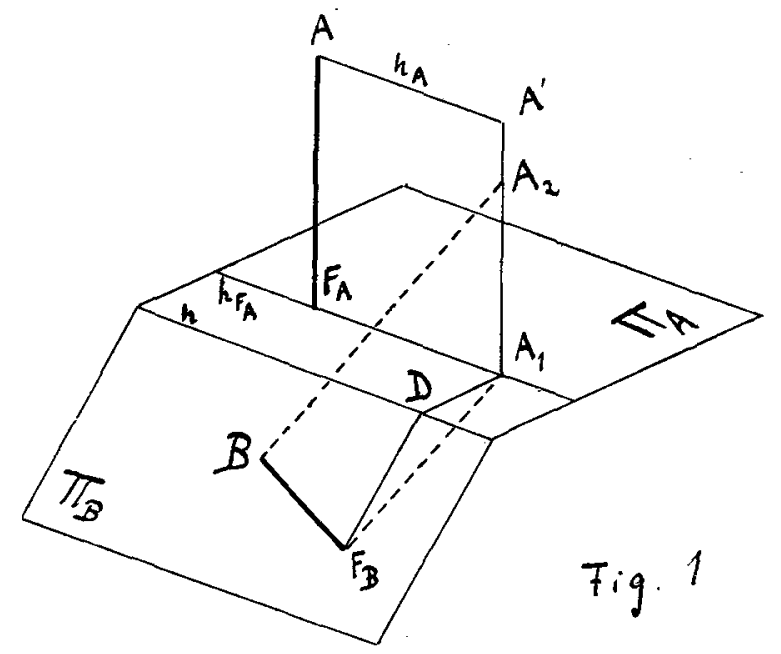

2. Die konvexe Fläche $\boldsymbol{\Phi}$ habe in $O$ eine Tangentialebene ${ }^{39}$, und $s$ sei ein schiefer ebener Schnitt von $\Phi$ durch $O$; die Ebene $\Pi(s)$ von $s$ bilde mit der Flächennormalen in $O$ den Winkel $\vartheta(s)$, mit $O<\vartheta(s)<\frac{\pi}{2}$. Den ron $\Pi(s)$ begrenzten offenen Halbraum, der die innere Normale von $\Phi$ in $O$ enthält, nennen wir die positive Seite ron $\Pi(s)$. Wir legen ein Koordinatensystem so, dass der Nullpunkt in $O$, die $x$-Achse in den Schnitt von $\pi(s)$ mit der Tangentialebene in $O$ und die innere Flächennormale in die positive $z$-Achse fällt; die positive $y$-Achse liege auf der positiven Seite von $\Pi(s)$.

so Die folgenden Überlegungen setzen teilweise voraus, dass diese Tangentialebene $\Phi$ nur in $O$ berührt; infolge mehrpunktiger Berührung evtl. nötig werdende Änderungen bestehen durchweg in Vereinfachungen. 
Die Ebene $\Pi(s)$ hat dann die Gleichung

$\Pi(s):$

$$
z=-y \cot \vartheta(s)
$$

Nun sei $\gamma(s)$ die Gerade mit den Gleichungen (vgl. Fig. 2)

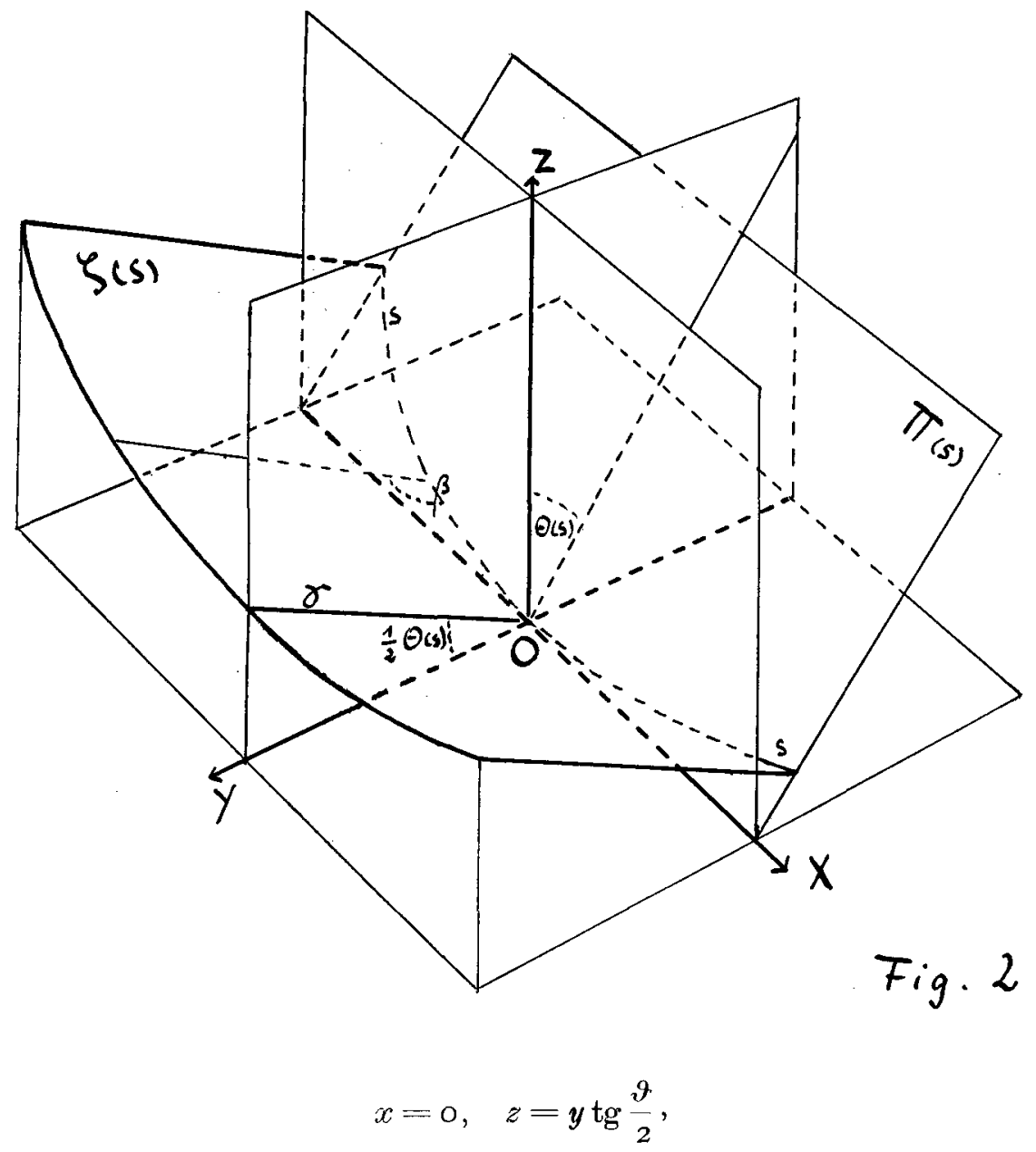

und $\zeta(s)$ der Zylinder durch den Schnitt $s$, dessen Erzeugenden parallel zu $\gamma(s)$ sind. Da die $x, y$-Ebene Tangentialebene im scharfen Sinne ist, definiert der Schnitt dieses Zylinders mit $\Phi$ je eine Halbumgebung auf $\zeta(s)$ und $\Phi$; erstere liegt ganz im Inneren von $\Phi$. Die offene Halbumgebung von $O$ auf $\Phi$ werde mit $h(s)$ bezeichnet. - Der Querschnitt des Zylinders ist eine konvexe, also rektifizierbare Kurve, so dass $\zeta(s)$ auf die Ebene abwickelbar ist. 
Hilfsatz 2. Auf $s$ gibt es auf beiden Seite von $O$ je einen Punkt $W_{i}(s)$, $i=\mathrm{I}, 2$, derart, dass der Bogen $\widehat{W_{1}(s) W_{9}}(s)$ nach Abyickelung des Zylinders $\zeta(s)$ eine konvexe Kurve wird, und zwar weisen ihre äusseren Normalen in der Umgebung von $O$ in das Innere von $h(s)$.

Beweis. Ist $\alpha$ der Winkel der Tangente bzw. Stützgeraden an $s$ mit der $x$-Achse, $\beta$ derjenige mit der Zylindererzeugenden, so ist

$$
\cos \beta=-\sin \alpha \sin \frac{\vartheta(s)}{2} .
$$

Im Punkte $O$ verschwindet $\alpha$. Wenn nun $\alpha$ von $-\frac{\pi}{2}$ bis $\frac{\pi}{2}$ läuft, so fällt $\cos \beta$ monoton; nach Abwickelung des Zylinders bleibt aber $\beta$ der Winkel mit den Zylindererzeugenden. Wählen wir also für $W_{i}(s)$ die beiden Punkte von $s$, in denen die Tangente bzw. Stützgerade senkrecht zur $x$-Achse steht, so erfüllt der Bogen ${\widehat{W_{1}(s) W_{2}}}_{2}(s)$ die Behauptung.

Hilfsatz 3. Zu jedem positiven $\delta$ gibt es eine Umgebung $u_{\delta}$ von $O$ auf $\Phi$, so dass die Punkte $W_{i}(s)$ für alle ebenen Schnitte $s$ mit $0<\vartheta(s)<\frac{\pi}{2}-\delta$ ausserhalb von $u_{\delta}$ liegen.

Beweis. Es sei $t(s)$ die Tangente von $s$ in $O$, und $l_{i}(s)$ das Lot von $W_{i}(s)$ auf $t(s)$. Wie aus dem vorigen Beweis hervorgeht, ist $l_{i}(s)$ zugleich Stützgerade an $s$, also auch an die Fläche $\Phi$, und bildet mit der $x, y$-Ebene den Winkel $\frac{\pi}{2}-\vartheta(s)>\delta$. Daher können die Berührungspunkte $W_{i}(s)$ von $l_{i}(s)$ mit der Fläche dem Punkte $O$ nicht beliebig nahe kommen.

Hilfsatz 4. $\mathrm{Zu}$ jedem positiven $\delta$ gibt es eine Umgebung $v_{\delta}$ von $O$ auf $\Phi$ derart, dass für alle ebenen Schnitte $s$ mit

$$
\delta<\vartheta(s)<\frac{\pi}{2}-\delta
$$

der auf der positiven Seite von $\Pi(s)$ liegende Teil von $v_{\delta}$ in $h(s)$ enthalten ist. (Das bedeutet eine Gleichmässigkeit der Ausdehnung der durch die Zylinder $\zeta(s)$ aus $\Phi$ ausgeschnittenen Halbumgebungen $h(s)$ von 0 .) 
Beweis. Wenn $v$ eine Umgebung von $O$ ist, für die der auf der positiven Seite von $\Pi(s)$ liegende Teil $v^{\prime}$ nicht in $h(s)$ enthalten ist, so hat $v^{\prime}$ Punkte mit der Begrenzung von $h(s)$ gemeinsam. Nun enthält $v^{\prime}$ nach Definition keine Punkte von $s$; es muss also auf $v^{\prime}$ Punkte ron $\Phi$ geben, in denen die Zylindererzengenden die Fläche zum zweiten male schneiden. Diese Schnittpunkte können aber dem Punkt $O$ nicht beliebig nahe kommen, d. h. es gibt eine Umgebung $v_{\delta}$ von $O$, die keine solchen Schnittpunkte enthält.

3. Es sei $\omega_{\delta}$ eine geodätische Kreisscheibe mit dem Mittelpunkt in $O$, die in den beiden Umgebungen $u_{\delta}$ und $v_{\delta}$ aus den Hilfsätzen 3 . und 4. enthalten ist. Jeder von $O$ ausgehende und in einem Punkte von $\omega_{\delta}$ endende kürzeste Bogen verläuft offenbar ganz in $\omega_{\delta}$. Wir zeigen nun zunächst:

Jeder in $\omega_{\delta}$ und auf der positiven Seite von $\Pi(s)$ verlaufende rektifizierbare Kurvenbogen $\tau$ dessen Endpunkte $T_{1}, T_{2}$ auf $s$ liegen, ist mindestens so lang wie der Bogen $\widehat{T}_{1} T_{2}$ auf $s$. (Dabei wird natürlich vorausgesetzt, dass $s$ der Beziehung (5) genügt.)

Zum Beweise betrachten wir den Ort der Fusspunkte von $\tau$ auf dem Zylinder $\zeta(s)$; er liegt ganz auf der positiven Seite von $\Pi(s)$ und ist höchstens so lang wie $\tau$ (Hilfsatz $\mathrm{I}$ ). Wir wickeln den Zylinder ab, und bezeichnen die Grössen nach der Abwicklung mit einem Strich. $s^{\prime}$ ist zwischen $T_{1}^{\prime}$ und $T_{2}^{\prime}$ konvex (Hilfsatz 3), und zwar verläuft $\boldsymbol{x}^{\prime}$ auf der äusseren Seite von $s^{\prime}$. Jeder auf dieser Seite verlaufende Bogen ist aber mindestens so lang, wie der Bogen $\widehat{T_{1}^{\prime} T_{2}^{\prime}}$ auf $s^{\prime}$, und da die Längen bei der Abwickelung erhalten bleiben, ist der Satz damit bewiesen.

Für die kürzesten Linien bedeutet der Satz folgendes:

I. Es sei $s$ irgend ein ebener Schnitt durch $O$, und $\delta$ so gewählt, dass (5) erfüllt ist. $R$ sei ein Punkt von $\omega_{\delta}$, der nicht auf der positiven Seite der Ebene $\Pi(s)$ liegt; dann kann kein kürzester Bogen, der von $O$ nach $R$ fïhrt, Punkte auf der positiven Seite von $\Pi(s)$ enthalten.

Nun schneidet jede von $O$ ausgehende Kurve, die dort keine Tangente hat, mindestens einen schiefen Schnitt $s$ durch $O$ unendlich oft (schief heisst: $\left.o<\vartheta(s)<\frac{\pi}{2}\right)$, d. h. die Kurve kommt in beliebiger Umgebung von $O$ unendlich oft auf die positive Seite von $\Pi(s)$. Demnach folgt aus I. unmittelbar:

II. Jede von $O$ ausgehende kürzeste Linie hat in $O$ eine Halbtangente. 
Wir beweisen nun:

III. Der von $O$ ausgehende kiirzeste Bogen $g$ habe in $O$ die Halbtangente $t$, und s sei ein schiefer Schnitt, der in 0 die Tangente $t$ hat. Ist dann (5) erfüllt so verläuft $g$ innerhalb der Umgebung $\omega_{\delta}$ ganz auf der positiven Seite von $\Pi(s)$.

Beweis. I) $g$ kann keinen Punkt von $\omega_{\delta}$ enthalten, der auf der negativen Seite von $\Pi(s)$ liegt (mit Ausschluss von $\Pi(s)$ selbst). Sonst betrachten wir den ebenen Sehnitt $s^{*}$ dureh $O$ und $R$, mit derselben Neigung $\mathscr{Y}\left(s^{*}\right)=\mathscr{Y}(s)$ in $O$ wie $s$ (es gibt deren allerdings zwei, doch nehmen wir den, für welchen der Winkel zwischen $\Pi(s)$ und $\Pi\left(s^{*}\right)$ klein wird). Die hinreichend nahe an $O$ gelegenen Punkté von $g$ liegen dann auf der positiven Seite von $\Pi\left(s^{*}\right)$, während $R$ anf $s^{*}$ liegt. Das widerspricht aber I.

2) $g$ enthält keinen Punkt von $s$. Sonst könnte nämlich nach I. $g$ keinen Punkt auf der positiven Seite von $\Pi(s)$ enthalten, und nach dem eben gesagten (III, I) auch nicht auf der negativen. $g$ müsste also zwischen $O$ und $R$ mit $s$ zusammenfallen. Dann gäbe es aber einen ebenen Schnitt $s^{*}$ durch $O$ und $R$ mit

$$
\vartheta(s)>\vartheta\left(s^{*}\right)>\delta
$$

Der Bogen zwischen $O$ und $R$ auf $s$ läge dann auf der positiven Seite von $\Pi\left(s^{*}\right)$, während $R$ auf $s^{*}$ liegt, was wiederum I. widerspricht.

IV. Jede von $O$ ausgehende kïrzeste Linie hat eine Schmiegebene, und diese geht durch die Flächennormale.

Es sei $\delta>0$ beliebig gewählt, und $s_{1}, s_{2}$ seien die beiden ebenen Schnitte durch die Tangente $t$ von $g$ in $O$, mit

$$
\vartheta\left(s_{i}\right)=2 \delta, \quad i=\mathrm{I}, 2 .
$$

Innerhalb der Umgebung $\omega_{\delta}$ verläuft dann $g$ nach III. ganz auf der positiven Seite sowohl von $\Pi\left(s_{1}\right)$, als auch ron $\Pi\left(s_{2}\right)$. Jede Ebene durch $t$ und einen Punkt, der auf der positiven Seite von $\Pi\left(s_{1}\right)$ und $\Pi\left(s_{2}\right)$ liegt, bildet aber mit der $z$-Achse höchstens den Winkel $2 \delta$. Sämtliche Grenzlagen der Ebenen durch $t$ und einen Punkt von $g$, der gegen $O$ konvergiert, bilden daher mit der $z$-Achse höchstens den Winkel $2 \delta$. Da aber $\delta$ beliebig klein gemacht werden kann, ist die Behauptung bewiesen.

Von den im Anfang dieses Paragraphen ausgesprochenen Sätzen bleibt also nur noch zu beweisen:

V. Jede durch $O$ gehende kürzeste Linie $g$ auf $\Phi$ hat in $O$ eine Tangente. 
Es seien $t$ und $t^{\prime}$ die rechte bzw. linke Halbtangente von $g$ in $O$, und $\left\{P_{n}\right\}$ und $\left\{P_{n}^{\prime}\right\}$ zwei Punktfolgen auf $g$, die von rechts bzw. links gegen $O$ konvergieren. Wenn $A$ und $B$ zwei Flächenpunkte sind, so bezeichne $l(A, B)$ die Entfernung ihrer Projektionen auf die Tangentialebene in $O, g(\dot{A}, B)$ ihre geodätische Entfernung. Dann ist offenbar

$$
\lim _{n \rightarrow \infty} \frac{g\left(P_{n}, P_{n}^{\prime}\right)}{l\left(P_{n}, P_{n}^{\prime}\right)}=\mathrm{I} .
$$

Andrerseits hat man

$$
\mathrm{I} \leq \frac{l\left(O, P_{n}\right)+l\left(O, P_{n}^{\prime}\right)}{l\left(P_{n}, P_{n}^{\prime}\right)} \leq \frac{g\left(O, P_{n}\right)+g\left(O, P_{n}^{\prime}\right)}{l\left(P_{n}, P_{n}^{\prime}\right)}=\frac{g\left(P_{n}, P_{n}^{\prime}\right)}{l\left(P_{n}, P_{n}^{\prime}\right)},
$$

und daher

$$
\lim _{n \rightarrow \infty} \frac{l\left(O, P_{n}\right)+l\left(O, P_{n}^{\prime}\right)}{l\left(P_{n}, P_{n}^{\prime}\right)}=\mathrm{I} .
$$

Hieraus folgt unmittelbar, dass der Winkel zwischen $t$ und $t^{\prime}$ verschwindet, d. h. dass die beiden Halbtangenten von $g$ in $O$ zusammenfallen.

Zusatz bei der Korrektur. Es sei darauf hingewiesen, dass sich aus den bewiesenen Sätzen nicht ableiten lässt, dass von einem Flächenpunkte mit Tangentialebene in jeder Richtung mindestens eine kürzeste Linie ausgeht. Das stimmt im allgemeinen auch garnicht, selbst dann nicht, wenn die Fläche überall eine Tangentialebene und eine Indikatrix besitzt. Dagegen stimmt die Behauptung für Flächen mit beschränkten oberen Normalkrümmungen. Für solche Flächen lassen sich auch Aussagen darüber machen, wie gross die Entfernung zweier Punkte sein muss, damit es zwischen ihnen mehr als eine kürzeste Verbindung gebe. -

Kopenhagen, den 27. April 1934. 